\title{
Effects of preoperative pulmonary function on short-term outcomes and overall survival after video-assisted thoracic surgery lobectomy
}

\author{
Yihe $\mathrm{Wu}^{1}$, Yuwei Zhou ${ }^{1}$, Shenhu Gao ${ }^{1}$, Chengli Du ${ }^{1}$, Linpeng $\mathrm{Yao}^{2}$, Rong Yang ${ }^{2}$ \\ ${ }^{1}$ Department of Thoracic Surgery, The First Affiliated Hospital, Zhejiang University School of Medicine, Hangzhou, China; ${ }^{2}$ Department of \\ Radiology, The First Affiliated Hospital, Zhejiang University School of Medicine, Hangzhou, China \\ Contributions: (I) Conception and design: R Yang; (II) Administrative support: Y Wu, R Yang; (III) Provision of study materials or patients: None; (IV) \\ Collection and assembly of data: Y Zhou, S Gao, C Du, L Yao; (V) Data analysis and interpretation: Y Wu; (VI) Manuscript writing: All authors; (VII) \\ Final approval of manuscript: All authors. \\ Correspondence to: Rong Yang, MD. Department of Radiology, The First Affiliated Hospital, Zhejiang University School of Medicine, \#79 Qingchun \\ Road, Hangzhou 310003, China. Email: dryangrong@zju.edu.cn.
}

Background: Preoperative pulmonary function tests are a necessary preoperative assessment tool for nonsmall cell lung cancer (NSCLC) patients awaiting surgery. We studied the effects of preoperative pulmonary function on short-term outcomes and overall survival (OS).

Methods: A retrospective cohort study was undertaken with adult NSCLC patients undergoing videoassisted thoracoscopic surgery (VATS) lobectomy between May 2016 and April 2017. The primary exposure variables were the percentage of predicted peak expiratory flow $(\mathrm{PEF} \%)$, the percentage of predicted forced vital capacity (FVC\%), and the percentage of predicted forced expiratory volume in $1 \mathrm{~s}$. The observation outcomes were postoperative pulmonary complications (PPCs), acute kidney injury (AKI), in-hospital mortality, readmission within 30 days, and OS. Univariate and multivariate analyses were performed.

Results: Of the 548 patients, postoperative pneumonia was observed in $206(37.6 \%)$. The results of the binary logistics regression analysis showed that relative to the moderate PEF\% group, the risk of postoperative pneumonia was significantly increased in the marginal PEF\% [odds ratio (OR) 2.076; 95\% confidence interval (CI): 1.211-3.558; P=0.008] and excellent PEF\% (OR 1.962; 95\% CI: 1.129-3.411; $\mathrm{P}=0.017$ ) groups. Relative to the good $\mathrm{FVC} \%$ group, the risk of postoperative pneumonia was significantly increased in the marginal FVC\% (OR 2.125; 95\% CI: 1.226-3.683; $\mathrm{P}=0.007)$ and moderate FVC\% (OR 2.230; 95\% CI: 1.298-3.832; $\mathrm{P}=0.004)$ groups. The OS analysis did not reveal any correlations among the pulmonary function parameters and OS in this cohort.

Conclusions: Preoperative PEF\% and FVC\% are associated with postoperative pneumonia in NSCLC patients undergoing VATS lobectomy. Preoperative $\mathrm{PEF} \%$ is as important as FVC\% in pulmonary function assessment before lung surgery.

Keywords: Pulmonary function; video-assisted thoracoscopic surgery (VATS); lobectomy; postoperative pneumonia

Submitted Aug 06, 2021. Accepted for publication Nov 12, 2021.

doi: $10.21037 / \mathrm{atm}-21-5244$

View this article at: https://dx.doi.org/10.21037/atm-21-5244 


\section{Introduction}

Lung cancer is the most common cause of cancer-related death worldwide (1). About $80 \%$ of lung cancers are Non-small cell lung cancer (NSCLC) (2). Lobectomy with systematic mediastinal lymph node dissection is the gold standard surgical treatment for early NSCLC (3). Preoperative pulmonary function assessment is the most basic assessment method for preoperative assessment of surgical tolerance, lung reserve, and risk of perioperative complications (2). Forced expiratory volume in $1 \mathrm{~s} \mathrm{(FEV1)}$ and forced vital capacity (FVC) are the most commonly used clinical pulmonary function parameters, which are useful for predicting perioperative complications, shortterm mortality, and long-term survival in NSCLC (3-6). However, research findings are controversial, and a unified consensus has yet to be reached $(2,7)$. Peak expiratory flow (PEF), which is defined as the maximum flow achieved during a forced expiration starting from the level of maximal lung inflation, is also an important pulmonary function parameter (8). However, apart from studies on FEV1 and FVC, few studies have analysed the effects of PEF on NSCLC patients following lobectomy.

In the past 30 years, video-assisted thoracoscopic surgery (VATS) has been widely used in thoracic surgery and is the standard surgical method for the treatment of lung cancer (9). Unlike traditional surgery, VATS can be performed through small incisions and results in less postoperative pain, a shorter hospital stay, and a more rapid recovery (1). However, evaluations of the relationship between preoperative pulmonary function and postoperative short- and longterm outcomes following VATS lobectomy in patients with NSCLC are limited. Thus, this retrospective study aimed to determine whether preoperative pulmonary functions are associated with postoperative outcomes in patients with NSCLC.

We focused on the effect of preoperative pulmonary functions including preoperative PEF, FVC, and FEV1 on the immediate and long-term post-surgical outcomes of patients with NSCLC undergoing VATS lobectomy to provide guidelines for the preoperative physiologic assessment of patients being considered for surgical resection of lung cancer.

We present the following article in accordance with the STROBE reporting checklist (available at https://dx.doi. org/10.21037/atm-21-5244).

\section{Methods}

\section{Patients}

This study is an observational retrospective study, including 592 consecutive NSCLC patients who underwent VATS lobectomy at The First Affiliated Hospital, College of Medicine, Zhejiang University, Hangzhou, China from May 1, 2016 to April 30, 2017. Patients were considered eligible for inclusion in the study if they were aged over 18 years and were underwent VATS lobectomy under general anaesthesia. The patients fasted for $8 \mathrm{~h}$ before surgery. A lung protective strategy (with low tidal volume) was adopted. The VATS lobectomy was performed through three ports. Systematic mediastinal lymph node dissection was performed. Patients with preoperative renal dysfunction, a history of thoracic surgery, congestive heart failure, or who required a sleeve lobectomy, a simultaneous resection of more than two lobes, or a second surgery were excluded from this study. The exclusion criteria for this study are detailed in Figure 1. Patients with missing pulmonary function data were also excluded. Ultimately, 548 patients were enrolled in the study (see Figure 1).

Demographics, intraoperative and postoperative data were all extracted from the patients' medical record system as described in Tables S1,S2. All patients received prophylactic first-generation/second-generation cephalosporins after surgery until the chest tubes were removed, but if the patient developed a postoperative lung infection, they were treated with sulbactam ampicillin or ciprofloxacin. In addition, if there was no risk of aspiration, patients usually started oral feedings $6-8$ hours after the tracheal intubation is extubated. The tumour-nodemetastasis classification (7th edition) proposed by the International Union Against Cancer was applied in this cohort.

The study was approved by the Medical Ethics Committee of the First Affiliated Hospital, Zhejiang University School of Medicine (No. 2017-58). As the data were recorded retrospectively and without any specific intervention, the Medical Ethics Committee waived informed consent. The study was conducted in accordance with Declaration of Helsinki (as revised in 2013).

\section{Exposure variables}

The exposure variables included the percentage of predicted 


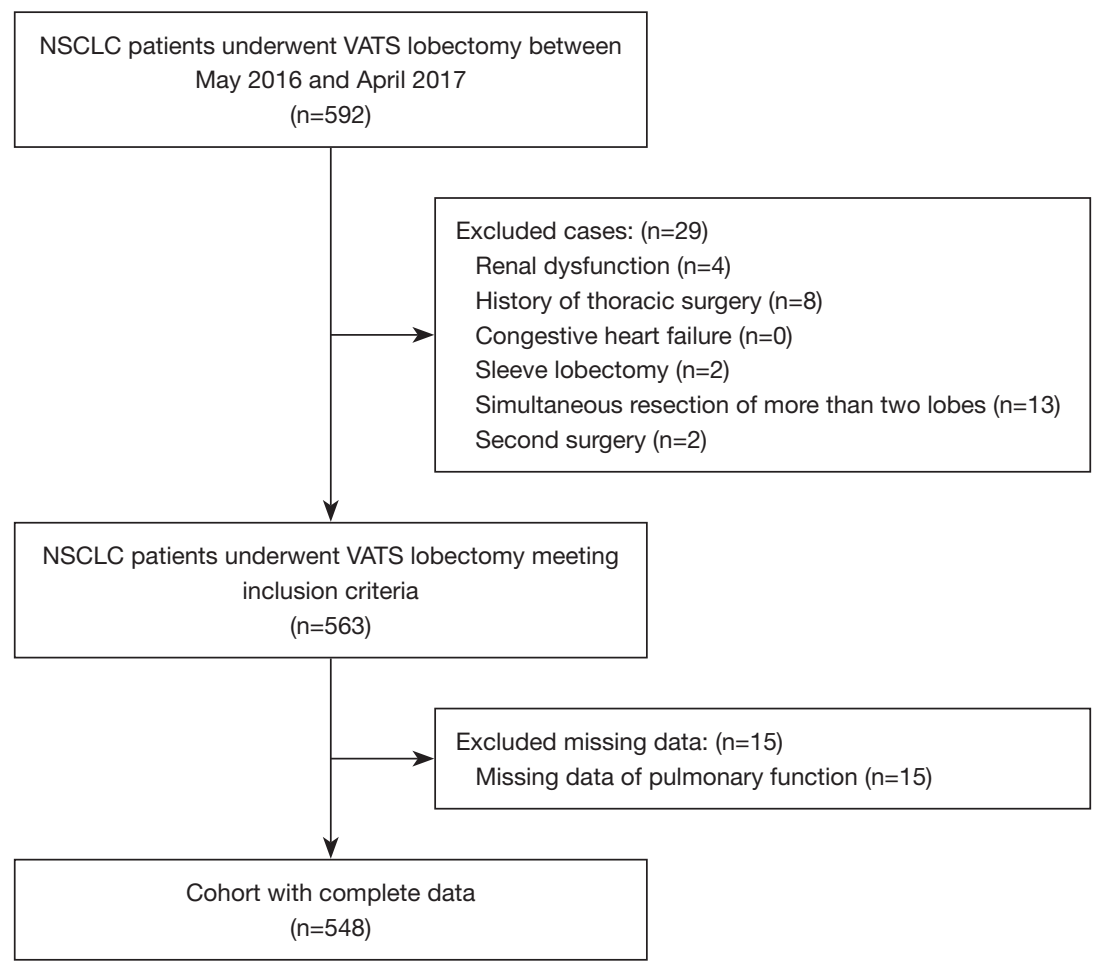

Figure 1 Study enrolment flow chart. NSCLC, non-small cell lung cancer; VATS, video-assisted thoracoscopic surgery.

$\mathrm{PEF}(\mathrm{PEF} \%)$, the percentage of predicted $\mathrm{FVC}(\mathrm{FVC} \%)$, and the percentage of predicted FEV1 (FEV1\%). To analyse the effect of these pulmonary function parameters on postoperative short-term outcomes and overall survival (OS), patients were classified into the following four groups representing incremental quartiles of the exposure variables of $\mathrm{PEF} \%, \mathrm{FVC} \%$, and $\mathrm{FEV} 1 \%$ : (I) marginal [quartile 1 (Q1)]; (II) moderate [quartile 2 (Q2)]; (III) good [quartile 3 (Q3)]; and (IV) excellent [quartile 4 (Q4)] (10).

\section{Short-term outcome measures}

The observation short-term outcome measures were postoperative pulmonary complications (PPCs), (including acute respiratory distress syndrome, reintubation, pulmonary embolism, the need for bedside bronchoscopy, prolonged air leak, failure to expand, atelectasis, and pneumonia during the period of postoperative hospitalisation), acute kidney injury (AKI), in-hospital mortality, and readmission within 30 days $(10,11)$. Pulmonary embolism needs to be diagnosed by computed tomography angiography of the pulmonary artery (12). Prolonged air leak was defined as the postoperative lung leakage time greater than 7 days
$(11,12)$. Failure to expand was defined as a pneumothorax with or without air leakage, which required chest X-ray diagnosis $(11,12)$. Atelectasis was diagnosed by chest radiograph documentation. The diagnosis of postoperative pneumonia needs to meet the following 3 conditions: a new pulmonary infiltrate on a chest $\mathrm{X}$-ray, leucocytosis and fever (ear temperature $\left.>37.5^{\circ} \mathrm{C}\right)(11,12)$. AKI was defined as the increase of serum creatinine level by more than $50 \%$ or $0.3 \mathrm{mg} / \mathrm{dL}$ compared with preoperative level within $48 \mathrm{~h}$ postoperatively or the existence of an AKI diagnostic code within 1 week postoperatively (10).

\section{OS measures}

All patients were followed up for OS, and the cause of death was extracted from patients' death certificate or medical records. We classified deaths into four categories, including deaths caused by postoperative complication during the initial hospital admission period or within 30 days after surgery, cancer-related deaths due to tumor progression/ recurrence, non-cancer-related deaths, and deaths with uncertain cause for those for whom records were not available. 


\section{Statistical analysis}

Data are expressed as mean \pm standard deviation for the continuous variables, and as a percentage for the categorical variables. The continuous data were compared using 1-way variance analysis. When the variance was not homogeneous, a non-parametric test (Kruskal-Wallis $\mathrm{H}$ test for multiple independent samples) was selected. $\mathrm{R} \times \mathrm{C}$ chi-square test was used for comparison of categorical variables. Fisher's exact test was used when the theoretical frequency was $<5$. Binary logistic regression analyses were conducted to determine the relationships between preoperative pulmonary function (PEF\%, FVC\%, and FEV1\%) and PPCs, AKI, inhospital mortality, and readmission within 30 days. Potential confounders were included based on a priori knowledge of predictors of PPCs, AKI, and OS (10). The assignment of the variables in the multivariate analysis is shown in Table $\mathrm{S} 3$. Odds ratios (ORs) with their $95 \%$ confidence intervals (CIs) were calculated from these models. Kaplan-Meier plots and log-rank tests were used to assess the effects of preoperative pulmonary function on survival. A multivariate Cox regression analysis was undertaken to estimate the hazard ratios and the $95 \%$ CIs for OS. A 2-tailed $\mathrm{P} \leq 0.05$ was considered statistically significant for all tests. All analyses were performed using SPSS 25.0 (SPSS, Inc., Chicago, IL, USA) $(10,12,13)$.

\section{Results}

\section{Patient selection and comparative univariate analysis}

Of the 548 patients who met the inclusion criteria (see Figure 1), 206 (37.6\%) cases of postoperative pneumonia were observed (see Table S2). No patient died during postoperative hospitalisation (see Table S2). The baseline characteristics and comparative univariate results of the cohort are set out in Tables S1,S2, and Tables S4-S7.

\section{Comparative multivariate analysis of 4 different $P E F \%$ groups}

All potential confounders were included in our multivariate regression model to determine the degree of contribution of the preoperative $\mathrm{PEF} \%$ on postoperative outcomes (see Table S8). Body mass index, instead of weight, was included in the regression model. Due to possible interactions among the various pulmonary function parameters, only the grouping variable PEF\% was included in the regression model. The binary logistics regression results revealed that the incidence of postoperative pneumonia was lowest in the moderate PEF\% (Q2) group (see Table 1 and Table S8). Conversely, the risk for postoperative pneumonia was significantly increased in the marginal PEF\% (Q1: OR 2.076; 95\% CI: 1.211-3.558; $\mathrm{P}=0.008$ ) and excellent PEF\% (Q4: OR 1.962; 95\% CI: 1.129-3.411; $\mathrm{P}=0.017$ ) groups (see Table 1). The incidences of other PPCs (including acute respiratory distress syndrome, reintubation, pulmonary embolism, the need for bedside bronchoscopy, prolonged air leak, failure to expand, and atelectasis) were similar among the four groups (see Table 1). The incidence of AKI was also similar among the four groups $(\mathrm{P}=0.168$; see Table 1). No deaths occurred among patients in the four groups during hospitalisation. There were also no statistical differences in readmission within 30 days among the four groups ( $\mathrm{P}=0.648$; see Table 1$)$.

\section{Comparative multivariate analysis of 4 different FVC\% groups}

All potential confounders were included in our multivariate regression model to determine the degree of contribution of preoperative FVC\% on postoperative outcomes (see Table S9). Patient weight and other pulmonary function parameters, including the PEF\%, FEV1\%, PEF (L/s), FVC (L), and FEV1 (L), were excluded from the regression model for the reason stated above. The binary logistics regression results affirmed that the incidence of postoperative pneumonia was lowest in the good (Q3) FVC\% group (see Table 2 and Table S9). Conversely, the postoperative risk of pneumonia was significantly increased in the marginal $\mathrm{FVC} \%(\mathrm{Q} 1$ : OR 2.125; 95\% CI: $1.226-3.683 ; \mathrm{P}=0.007)$ and moderate $\mathrm{FVC} \%$ (Q2: OR 2.230; 95\% CI: 1.298-3.832; $\mathrm{P}=0.004)$ groups (see Table 2). The incidence of other PPCs (including acute respiratory distress syndrome, reintubation, pulmonary embolism, the need for bedside bronchoscopy, prolonged air leak, failure to expand, and atelectasis) were similar among the four groups (see Table 2). The incidence of AKI was also similar among the four groups $(\mathrm{P}=0.439$; see Table 2). No deaths occurred among patients in the four groups during hospitalisation. Readmission within 30 days was also comparable among the four groups $(\mathrm{P}=0.802$; see Table 2$)$.

\section{Comparative multivariate analysis of 4 different FEV $1 \%$ groups}

All potential confounders were included in our multivariate regression model to determine the degree of contribution 
Table 1 Effects of the PEF\% on postoperative outcomes

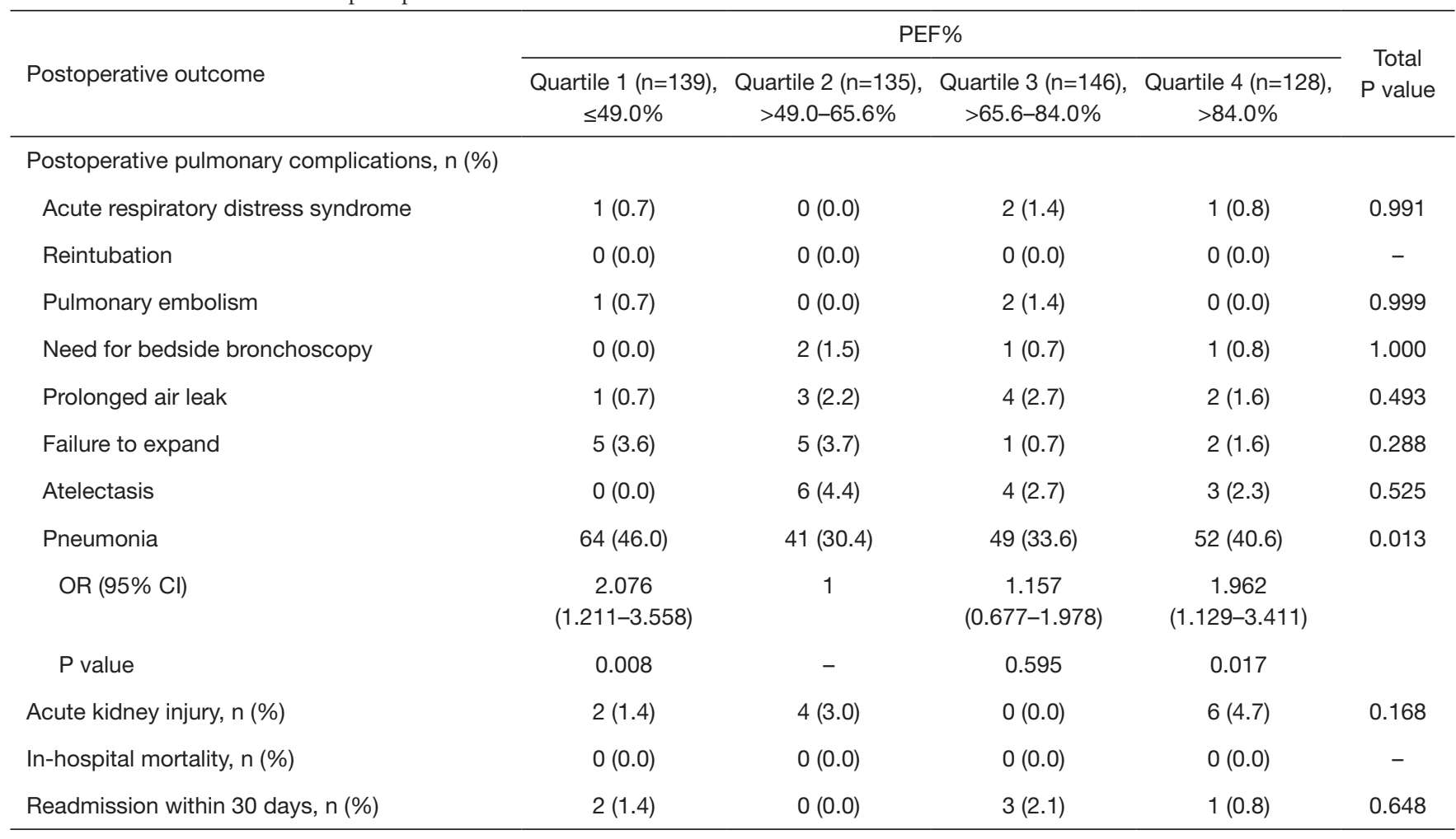

The results of the binary logistics regression are presented as the adjusted $\mathrm{OR}, 95 \% \mathrm{Cl}$, and $\mathrm{P}$ value. The best-performing quartile 2 served as the reference group. $\mathrm{Cl}$, confidence interval; OR, odds ratio; PEF\%, peak expiratory flow as a percentage of predicted.

of the preoperative $\mathrm{FEV} 1 \%$ on postoperative outcomes (see Table S10). The binary logistics regression results showed that the incidence of all PPCs (including acute respiratory distress syndrome, reintubation, pulmonary embolism, the need for bedside bronchoscopy, prolonged air leak, failure to expand, atelectasis, and pneumonia) were similar among the four different FEV1\% groups (see Table 3 and Table S10). The incidence of AKI was also similar among the four groups ( $\mathrm{P}=0.964$; see Table 3). No deaths occurred among patients in the four groups during hospitalisation. There were also no statistical differences in readmission within 30 days among the four groups ( $\mathrm{P}=0.812$; see Table 3).

\section{OS analysis}

The median follow-up period for all patients was 28.7 months. Nine (1.7\%) deaths had occurred at the median follow-up period of 13.6 months. Causes of death included early postoperative complications $(0.0 \%)$, cancer-related deaths $(55.6 \%)$, non-cancer-related deaths $(0.0 \%)$, and cause of death uncertain (44.4\%) (see Table 4 and Tables S11,S12).
There were no statistical differences in OS among the 4 different PEF\%, FVC\%, and FEV1\% groups (see Figure 2), and these findings were further confirmed by the multivariate Cox regression results (see Tables S13-S15).

In addition, we grouped the patients using the absolute values of preoperative PEF, FVC, and FEV1 as grouping variables. Patients were also classified into 4 groups representing incremental quartiles of the exposure variables of PEF (L/s), FVC (L), and FEV1 (L). The baseline characteristics and comparative univariate results of the cohort are presented in Tables S16-S21. The results of the multivariate analysis and OS analysis are presented in Tables S22-S33, and Figure S1.

\section{Discussion}

In the analysis of the 548 NSCLC patients who underwent VATS lobectomy, a robust association between preoperative pulmonary functions (PEF\% and FVC\%) and postoperative pneumonia was observed (see Tables 1,2). However, this association did not lead to differences in OS (see Figure 2). 
Table 2 Effects of the FVC\% on postoperative outcomes

\begin{tabular}{|c|c|c|c|c|c|}
\hline Postoperative outcome & \multicolumn{4}{|c|}{ FVC\% } & $\begin{array}{c}\text { Total } \\
\text { P value }\end{array}$ \\
\hline Acute respiratory distress syndrome & $2(1.4)$ & $0(0.0)$ & $1(0.8)$ & $1(0.7)$ & 0.959 \\
\hline Need for bedside bronchoscopy & $0(0.0)$ & $2(1.5)$ & $2(1.5)$ & $0(0.0)$ & 0.842 \\
\hline Prolonged air leak & $2(1.4)$ & $1(0.7)$ & $3(2.3)$ & $4(3.0)$ & 0.755 \\
\hline Failure to expand & $2(1.4)$ & $6(4.4)$ & $0(0.0)$ & $5(3.7)$ & 0.616 \\
\hline$P$ value & 0.007 & 0.004 & - & 0.237 & \\
\hline Acute kidney injury, n (\%) & $4(2.8)$ & $3(2.2)$ & $0(0.0)$ & $5(3.7)$ & 0.439 \\
\hline In-hospital mortality, n (\%) & $0(0.0)$ & $0(0.0)$ & $0(0.0)$ & $0(0.0)$ & - \\
\hline Readmission within 30 days, $\mathrm{n}(\%)$ & $1(0.7)$ & $0(0.0)$ & $2(1.5)$ & $3(2.2)$ & 0.802 \\
\hline
\end{tabular}

The results of the binary logistics regression are presented as the adjusted $\mathrm{OR}, 95 \% \mathrm{Cl}$, and $\mathrm{P}$ value. The best-performing quartile 3 served as the reference group. $\mathrm{Cl}$, confidence interval; FVC\%, forced vital capacity as percentage of predicted; OR, odds ratio.

Neither postoperative pneumonia nor OS was significantly associated with the FEV1\%. The major finding of this study is that for NSCLC patients who underwent VATS lobectomy, a preoperative $\mathrm{PEF} \%$ value that was too low $(\leq 49.0 \%)$ or too high $(>84.0 \%)$ led to an increased incidence of postoperative pneumonia (see Table 1). Conversely, only a preoperative $\mathrm{FVC} \%$ value that was too low $(\leq 92.0 \%)$ led to an increased incidence of postoperative pneumonia (see Table 2). Thus, preoperative PEF\% is as important as $\mathrm{FVC} \%$ in pulmonary function assessment before lung surgery.

To estimate the risk of complications, including pneumonia and atelectasis, FEV1 and FVC have been studied in the last quarter of the 1900s (14-17). FEV1 and FVC have traditionally been considered the two critical pulmonary function parameters for lung cancer candidates awaiting surgery $(8,18,19)$. FEV1 is a measure of how much air can be exhaled in $1 \mathrm{~s}$ following a deep inhalation. No significant association was found between FEV1\% and postoperative pneumonia in the current study. FVC is a measurement of lung size (in litres) and represents the volume of air in the lungs that can be exhaled following a deep inhalation. Historically, research has focused on the lower threshold of a $\mathrm{FVC} \%$ for curative lung resection, but the upper threshold of a $\mathrm{FVC} \%$ of $60 \%$ has not received much attention (20). Under the conventional practice specification at our hospital, only patients with a $\mathrm{FVC} \%$ $>60 \%$ can undergo a lobectomy. The average value of $\mathrm{FVC} \%$ in this group was $91.3 \% \pm 15.7 \%$ (see Table S1). However, while the $\mathrm{FVC} \%$ was screened before surgery, patients with a marginal and moderate $\mathrm{FVC} \%(\leq 92.0 \%)$ had an increased incidence of postoperative pneumonia. The main cause of postoperative pneumonia is the decrease of cough ability and sputum excretion ability caused by surgical trauma and postoperative decline of pulmonary function (18). After lobectomy, a fall in the FEV $1 \%$ from $12 \%$ to $23 \%$, and a fall in the $\mathrm{FVC} \%$ from $10 \%$ to $30 \%$ has been reported (21). The lower the preoperative lung function, the more significant the decline in postoperative lung function (21). For patients with an impaired preoperative $\mathrm{FVC} \%$, combinations of bronchodilators, physical therapy, smoking cessation, and corticosteroids might be needed to reduce the risk of postoperative respiratory complications (22).

PEF refers to a person's maximum speed of expiration. 
Table 3 Effects of the FEV1\% on postoperative outcomes

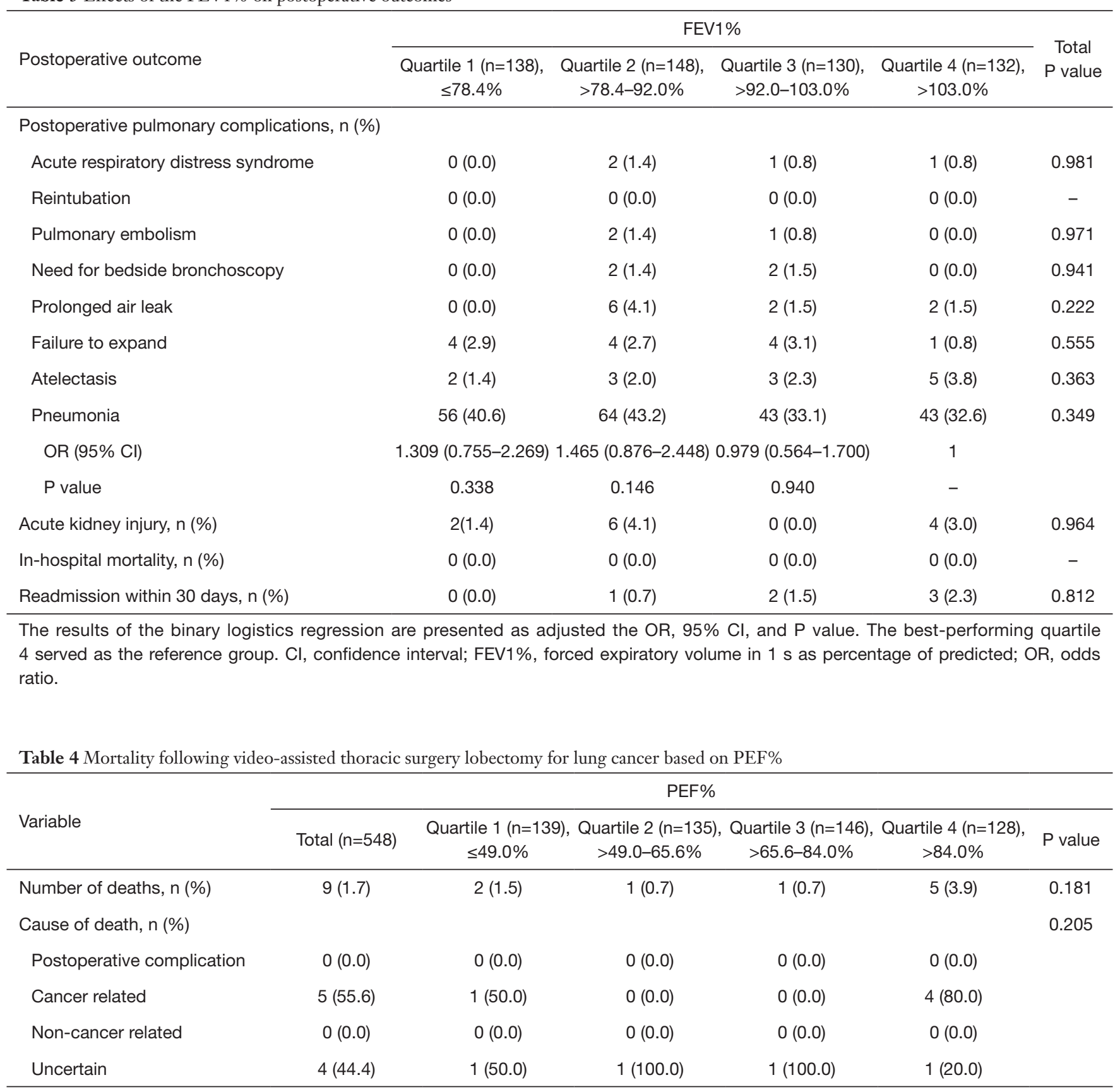

Values are presented as $\mathrm{n}(\%)$. PEF\%, peak expiratory flow as a percentage of predicted.

It measures the airflow through the bronchi and thus the degree of obstruction in the airways. In clinical practice, $\mathrm{PEF}$ is rarely used to assess the risk of complications associated with lung resection. In recent years, several studies have found that PEF is closely related to cardiovascular events and lung cancer mortality, and is an important indicator of physical functioning and health status in the elderly population (8,23-26). PEF reflects airway patency and resistance, and respiratory muscle strength and thus reflects a patient's cough and expectoration ability, which is especially important for patients undergoing lung resection (8). In our study, we observed that a preoperative 

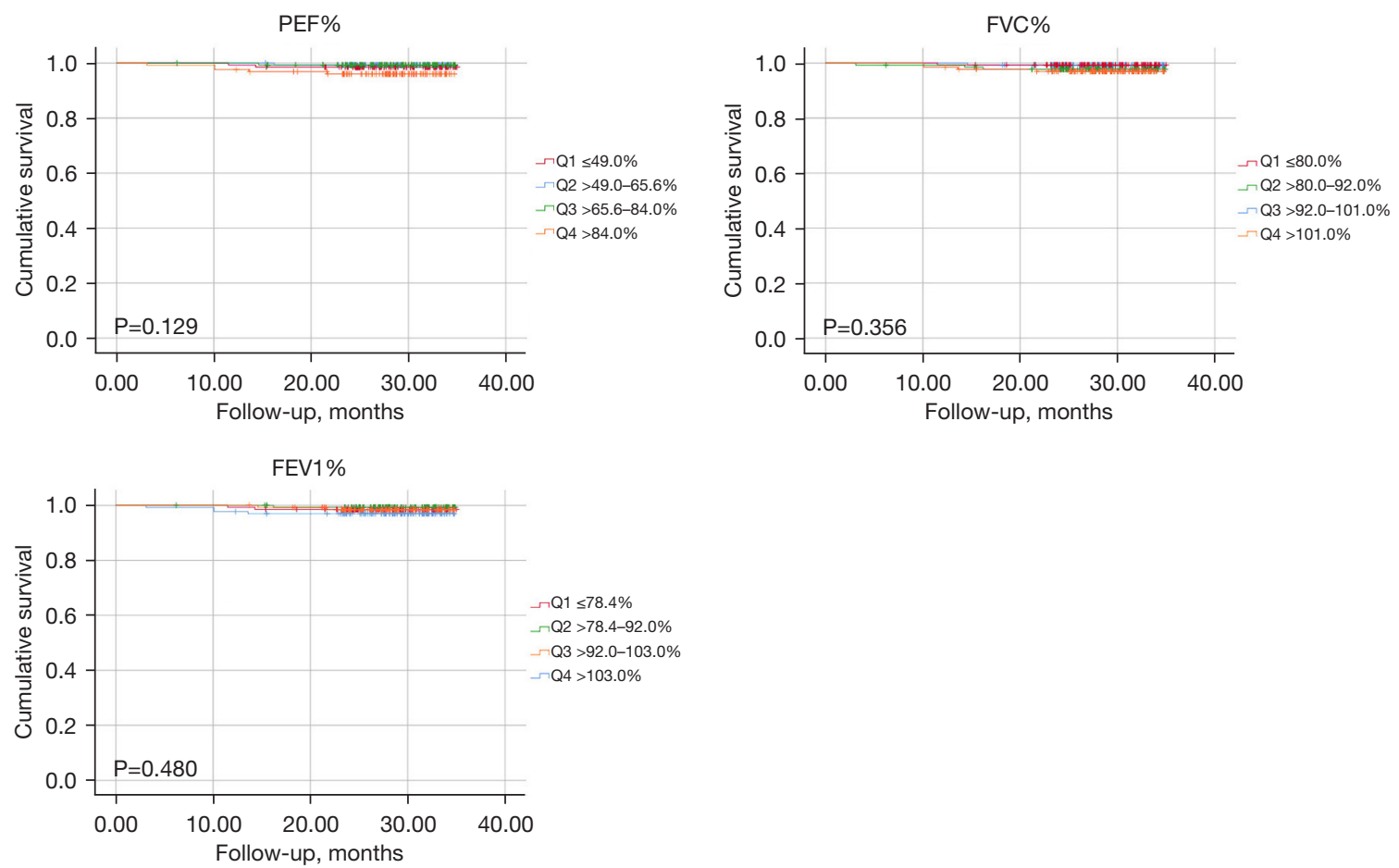

Figure 2 Overall survival curves in 548 patients among the 4 different groups according to the predicted pulmonary function values of $\mathrm{PEF} \%, \mathrm{FVC} \%$, and $\mathrm{FEV} 1 \%$. FEV1\%, forced expiratory volume in $1 \mathrm{~s}$ as a percentage of predicted; FVC\%, forced vital capacity as a percentage of predicted; PEF\%, peak expiratory flow as a percentage of predicted; Q1-Q4, quartiles 1 to 4.

PEF\% that was too low $(\leq 49.0 \%)$ or too high $(>84.0 \%)$ led to an increased incidence of postoperative pneumonia in NSCLC patients undergoing VATS lobectomy (see Table 1). A PEF\% that was too low caused an increase in the incidence of postoperative pneumonia due to the poor cough and expectoration ability of the patients. However, the reasons for the increase in the incidence of postoperative pneumonia in patients with a $\mathrm{PEF} \%$ that was too high are difficult to reasonably explain, and currently, no other studies appear to have examined this issue. Thus, further research is required. There is good evidence that PEF, which can be measured rapidly and easily with an inexpensive and hand-held device, is both reliable and reproducible (27). Further, PEF is more reproducible than FEV1 (27). Thus, the PEF\% should be considered as a primary pulmonary function parameter for the preoperative assessment of NSCLC patients awaiting surgery.

In relation to the other PPCs (including acute respiratory distress syndrome, reintubation, pulmonary embolism, the need for bedside bronchoscopy, prolonged air leak, failure to expand, and atelectasis), we did not find any associations among the various parameters of pulmonary function examined. In addition, other shortterm outcomes (including AKI, in-hospital mortality, and readmission within 30 days) were also not associated with the pulmonary function parameters. The OS analysis (for which there was a median follow-up period of 28.7 months) showed no correlation between the pulmonary function parameters and OS in this cohort of NSCLC patients. Similarly, Almquist et al. noted that after a median follow-up period of 53.2 months, preoperative pulmonary function did not predict survival in resected early-stage NSCLC patients (4).

\section{Limitations}

The present study had several limitations. First, as a single-centre retrospective study (the study population predominantly comprised patients who were deemed good surgical candidates), it carries the inherent possibility of selection bias. Second, due to its retrospective nature, any diagnosis of postoperative pneumonia lacked pathogenic evidence. However, our diagnostic criteria for postoperative pneumonia (a new pulmonary infiltrate on a chest X-ray 
with leucocytosis and fever) are objective and reproducible. Third, because the proportion of IA-stage NSCLC patients in this cohort was as high as $81.6 \%$, the median followup period of 28.7 months was slightly insufficient, and the evaluation of OS was slightly inefficient. Fourth, our study did not include the diffusion capacity of carbon monoxide (DLCO) of pulmonary function in the analysis. As the preoperative DLCO assessment at our centre is not mandatory and is usually replaced by arterial oxygen pressure, DLCO data were only available for 145 of 548 patients in this cohort. However, despite these limitations, a relatively homogeneous population, treated uniformly at a single centre, was analysed. Thus, we believe that our data are valid.

\section{Conclusions}

In summary, the preoperative $\mathrm{PEF} \%$ and $\mathrm{FVC} \%$ are associated with postoperative pneumonia in NSCLC patients undergoing VATS lobectomy, but this association does not necessarily lead to differences in OS. We found that a preoperative PEF\% that was too low $(\leq 49.0 \%)$ or too high $(>84.0 \%)$ led to an increased incidence of postoperative pneumonia; however, only a preoperative $\mathrm{FVC} \%$ that was too low $(\leq 92.0 \%)$ led to an increased incidence of postoperative pneumonia. An increase in the application of the $\mathrm{PEF} \%$ in preoperative assessment in NSCLC patients awaiting surgery might prevent postoperative pneumonia.

\section{Acknowledgments}

The authors appreciate the assistance of the Large-Scale Data Analysis Centre of Cancer Precision MedicineLinkDoc database from where the clinical and pathological data were collected.

Funding: This work was supported by the National Natural Science Foundation of China, China (grant number 31700690), and the Natural Science Foundation of Zhejiang Province, China (grant number LQ18H180002).

\section{Footnote}

Reporting Checklist: The authors have completed the STROBE reporting checklist. Available at https://dx.doi. org/10.21037/atm-21-5244

Data Sharing Statement: Available at https://dx.doi. org/10.21037/atm-21-5244
Conflicts of Interest: All authors have completed the ICMJE uniform disclosure form (available at https://dx.doi. org/10.21037/atm-21-5244). The authors have no conflicts of interest to declare.

Ethical Statement: The authors are accountable for all aspects of the work in ensuring that questions related to the accuracy or integrity of any part of the work are appropriately investigated and resolved. The study was approved by the Medical Ethics Committee of the First Affiliated Hospital, Zhejiang University School of Medicine (No. 2017-58). As the data were recorded retrospectively and without any specific intervention, the Medical Ethics Committee waived informed consent. The study was conducted in accordance with the Declaration of Helsinki (as revised in 2013).

Open Access Statement: This is an Open Access article distributed in accordance with the Creative Commons Attribution-NonCommercial-NoDerivs 4.0 International License (CC BY-NC-ND 4.0), which permits the noncommercial replication and distribution of the article with the strict proviso that no changes or edits are made and the original work is properly cited (including links to both the formal publication through the relevant DOI and the license). See: https://creativecommons.org/licenses/by-nc$\mathrm{nd} / 4.0 /$.

\section{References}

1. Kim SJ, Lee YJ, Park JS, et al. Changes in pulmonary function in lung cancer patients after video-assisted thoracic surgery. Ann Thorac Surg 2015;99:210-7.

2. Gao Y, Zhang H, Li Y, et al. Preoperative pulmonary function correlates with systemic inflammatory response and prognosis in patients with non-small cell lung cancer: results of a single-institution retrospective study. Oncotarget 2017;8:27489-501.

3. Chen C, Ni Q, Shi Y, et al. Prognosis analysis of lobectomy and sublobar resection in pa-tients $\geq 75$ years old with pathological stage I invasive lung adenocarcinoma of $\leq 3 \mathrm{~cm}$ : a propensity score matchingbased analysis. Transl Cancer Res 2019;8:574-82.

4. Almquist D, Khanal N, Smith L, et al. Preoperative Pulmonary Function Tests (PFTs) and Outcomes from Resected Early Stage Non-small Cell Lung Cancer (NSCLC). Anticancer Res 2018;38:2903-7.

5. Bertani A, Ferrari PA, De Monte L, et al. Video-assisted 
thoracic surgery lobectomy in patients with reduced pulmonary function: a single-center series. Future Oncol 2016;12:39-45.

6. Garzon JC, Ng CS, Sihoe AD, et al. Video-assisted thoracic surgery pulmonary resection for lung cancer in patients with poor lung function. Ann Thorac Surg 2006;81:1996-2003.

7. Guo X, Cao H, Xu J, et al. Forced vital capacity predicts long-term survival for curative-resected NSCLC. Med Oncol 2014;31:146.

8. Lai Y, Wang X, Li P, et al. Preoperative peak expiratory flow (PEF) for predicting postoperative pulmonary complications after lung cancer lobectomy: a prospective study with 725 cases. J Thorac Dis 2018;10:4293-301.

9. Lewis RJ, Caccavale RJ, Sisler GE, et al. One hundred consecutive patients undergoing video-assisted thoracic operations. Ann Thorac Surg 1992;54:421-6.

10. Shin CH, Long DR, McLean D, et al. Effects of Intraoperative Fluid Management on Postoperative Outcomes: A Hospital Registry Study. Ann Surg 2018;267:1084-92.

11. Arslantas MK, Kara HV, Tuncer BB, et al. Effect of the amount of intraoperative fluid administration on postoperative pulmonary complications following anatomic lung resections. J Thorac Cardiovasc Surg 2015;149:31420, 321.e1.

12. Wu Y, Yang R, Xu J, et al. Effects of Intraoperative Fluid Management on Postoperative Outcomes After Lobectomy. Ann Thorac Surg 2019;107:1663-9.

13. Lugg ST, Agostini PJ, Tikka T, et al. Long-term impact of developing a postoperative pulmonary complication after lung surgery. Thorax 2016;71:171-6.

14. Ali MK, Mountain CF, Ewer MS, et al. Predicting loss of pulmonary function after pulmonary resection for bronchogenic carcinoma. Chest 1980;77:337-42.

15. Nakahara K, Ohno K, Hashimoto J, et al. Prediction of postoperative respiratory failure in patients undergoing lung resection for lung cancer. Ann Thorac Surg 1988;46:549-52.

16. Putnam JB Jr, Lammermeier DE, Colon R, et al. Predicted pulmonary function and survival after pneumonectomy for primary lung carcinoma. Ann Thorac Surg 1990;49:90914; discussion 915.

17. Yokoba M, Ichikawa T, Harada S, et al. Postoperative pulmonary function changes according to the resected lobe: a 1-year follow-up study of lobectomized patients. J Thorac Dis 2018;10:6891-902.
18. Agostini PJ, Lugg ST, Adams K, et al. Risk factors and short-term outcomes of postoperative pulmonary complications after VATS lobectomy. J Cardiothorac Surg 2018;13:28.

19. Wasswa-Kintu S, Gan WQ, Man SF, et al. Relationship between reduced forced expiratory volume in one second and the risk of lung cancer: a systematic review and metaanalysis. Thorax 2005;60:570-5.

20. Ozeki N, Kawaguchi K, Okasaka T, et al. Marginal pulmonary function is associated with poor short- and long-term outcomes in lung cancer surgery. Nagoya J Med Sci 2017;79:37-42.

21. Drakou E, Kanakis MA, Papadimitriou L, et al. Changes in Simple Spirometric Parameters After Lobectomy for Bronchial Carcinoma. J Cardiovasc Thorac Res 2015;7:68-71.

22. Lee JY, Jin SM, Lee CH, et al. Risk factors of postoperative pneumonia after lung cancer surgery. J Korean Med Sci 2011;26:979-84.

23. Fragoso CA, Gahbauer EA, Van Ness PH, et al. Peak expiratory flow as a predictor of subsequent disability and death in community-living older persons. J Am Geriatr Soc 2008; 56:1014-20.

24. Hegewald MJ, Lefor MJ, Jensen RL, et al. Peak expiratory flow is not a quality indicator for spirometry: peak expiratory flow variability and FEV1 are poorly correlated in an elderly population. Chest 2007;131:1494-9.

25. Roberts MH, Mapel DW. Limited lung function: impact of reduced peak expiratory flow on health status, healthcare utilization, and expected survival in older adults. Am J Epidemiol 2012;176:127-34.

26. Vaz Fragoso CA, Gahbauer EA, Van Ness PH, et al. Reporting peak expiratory flow in older persons. J Gerontol A Biol Sci Med Sci 2007;62:1147-51.

27. White P. Spirometry and peak expiratory flow in the primary care management of COPD. Prim Care Respir J 2004;13:5-8.

(English Language Editor: L. Huleatt)

Cite this article as: $\mathrm{Wu} \mathrm{Y,} \mathrm{Zhou} \mathrm{Y,} \mathrm{Gao} \mathrm{S,} \mathrm{Du} \mathrm{C,} \mathrm{Yao} \mathrm{L,}$ Yang R. Effects of preoperative pulmonary function on shortterm outcomes and overall survival after video-assisted thoracic surgery lobectomy. Ann Transl Med 2021;9(22):1651. doi: 10.21037/atm-21-5244 

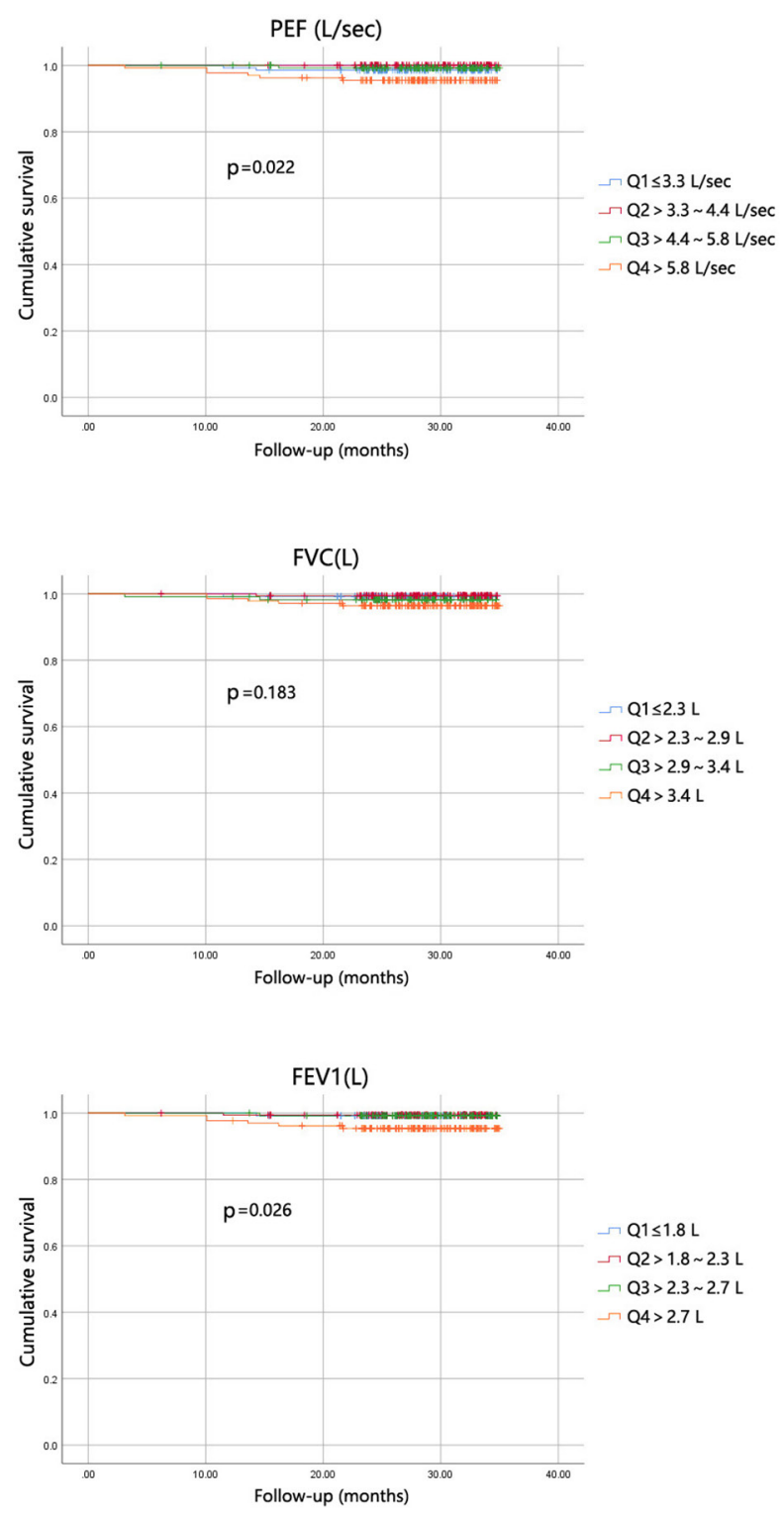

Figure S1 Overall survival curves in 548 patients among the 4 different groups according to the absolute pulmonary function values of PEF (L/s), FVC (L), and FEV1 (L). FEV1, forced expiratory volume in $1 \mathrm{~s}$; FVC, forced vital capacity; PEF, peak expiratory flow; Q1-Q4, quartiles 1 to 4. 
Table S1 Clinical characteristics of patients based on PEF\%

\begin{tabular}{|c|c|c|c|c|c|c|}
\hline \multirow{3}{*}{ Characteristic } & \multicolumn{6}{|c|}{ PEF\% } \\
\hline & \multirow{3}{*}{ Total $(n=548)$} & \multirow{2}{*}{\multicolumn{4}{|c|}{$\begin{array}{l}\text { Quartile } 1(n=139) \text {, Quartile } 2(n=135) \text {, Quartile } 3(n=146) \text {, Quartile } 4(n=128) \text {, } \\
\qquad 49.0 \%>49.0-65.6 \%>65.6-84.0 \% \quad>84.0 \%\end{array}$}} & \multirow{2}{*}{$P$ value } \\
\hline & & & & & & \\
\hline ASA score & & & & & & 0.084 \\
\hline I & $459(83.8 \%)$ & $110(79.1 \%)$ & $118(87.4 \%)$ & $126(86.3 \%)$ & $105(82.0 \%)$ & \\
\hline II & $66(12.0 \%)$ & $18(12.9 \%)$ & $11(8.1 \%)$ & $17(11.6 \%)$ & $20(15.6 \%)$ & \\
\hline III & $23(4.2 \%)$ & $11(7.9 \%)$ & $6(4.4 \%)$ & $3(2.1 \%)$ & $3(2.3 \%)$ & \\
\hline Age & $63.0 \pm 10.6$ & $65.0 \pm 9.7$ & $63.5 \pm 10.4$ & $63.5 \pm 10.3$ & $59.6 \pm 11.4$ & 0.000 \\
\hline Gender (female/male) & $331 / 217$ & $71 / 68$ & $85 / 50$ & $92 / 54$ & $83 / 45$ & 0.075 \\
\hline Weight, kg & $61.4 \pm 9.8$ & $62.8 \pm 10.4$ & $61.4 \pm 9.8$ & $61.5 \pm 9.6$ & $59.7 \pm 9.1$ & 0.081 \\
\hline BMI, $\mathrm{kg} / \mathrm{m}^{2}$ & $23.2 \pm 2.8$ & $23.5 \pm 2.7$ & $23.3 \pm 3.2$ & $23.2 \pm 2.9$ & $22.6 \pm 2.5$ & 0.043 \\
\hline Smoking & $146(26.6 \%)$ & $56(40.3 \%)$ & $30(22.2 \%)$ & $33(22.6 \%)$ & $27(21.1 \%)$ & 0.000 \\
\hline Diabetes mellitus & $48(8.8 \%)$ & $17(12.2 \%)$ & $7(5.2 \%)$ & $12(8.2 \%)$ & $12(9.4 \%)$ & 0.224 \\
\hline Coronary heart disease & $9(1.6 \%)$ & $4(2.9 \%)$ & $2(1.5 \%)$ & $2(1.4 \%)$ & $1(0.8 \%)$ & 0.594 \\
\hline FEV1, L & $2.3 \pm 0.6$ & $2.0 \pm 0.5$ & $2.2 \pm 0.6$ & $2.4 \pm 0.6$ & $2.6 \pm 0.6$ & 0.000 \\
\hline FVC, L & $2.9 \pm 0.7$ & $2.7 \pm 0.7$ & $2.8 \pm 0.7$ & $3.0 \pm 0.8$ & $3.2 \pm 0.7$ & 0.000 \\
\hline PEF, L/s & $4.7 \pm 1.9$ & $2.7 \pm 0.7$ & $3.9 \pm 0.8$ & $5.2 \pm 1.1$ & $7.0 \pm 1.5$ & 0.000 \\
\hline FEV1\% & $(91.1 \pm 17.2) \%$ & $(76.5 \pm 12.8) \%$ & $(87.4 \pm 14.7) \%$ & $(97.3 \pm 15.7) \%$ & $(103.6 \pm 11.9) \%$ & 0.000 \\
\hline FVC\% & $(91.3 \pm 15.7) \%$ & $(83.1 \pm 13.2) \%$ & $(87.5 \pm 15.0) \%$ & $(94.8 \pm 15.6) \%$ & $(100.1 \pm 13.2) \%$ & 0.000 \\
\hline PEF\% & $(67.6 \pm 24.0) \%$ & $(39.8 \pm 6.4) \%$ & $(57.2 \pm 4.9) \%$ & $(74.5 \pm 5.4) \%$ & $(101.1 \pm 15.0) \%$ & 0.000 \\
\hline Intraoperative bleeding, mL & $47.2 \pm 32.0$ & $53.6 \pm 43.3$ & $42.7 \pm 25.8$ & $50.0 \pm 32.2$ & $41.7 \pm 19.4$ & 0.097 \\
\hline Intraoperative blood transfusion, $\mathrm{mL}$ & 0 & 0 & 0 & 0 & 0 & - \\
\hline Length of operation, min & $132.6 \pm 35.6$ & $131.3 \pm 38.6$ & $130.5 \pm 33.2$ & $135.8 \pm 36.4$ & $132.5 \pm 33.9$ & 0.618 \\
\hline NSCLC staging & & & & & & 0.042 \\
\hline IA & 447 (81.6\%) & $115(82.7 \%)$ & $103(76.3 \%)$ & $120(82.2 \%)$ & $109(85.2 \%)$ & \\
\hline IB & $37(6.8 \%)$ & $6(4.3 \%)$ & $13(9.6 \%)$ & $12(8.2 \%)$ & $6(4.7 \%)$ & \\
\hline IIA & $29(5.3 \%)$ & $10(7.2 \%)$ & $7(5.2 \%)$ & $10(6.8 \%)$ & $2(1.6 \%)$ & \\
\hline IIB & $2(0.4 \%)$ & $0(0.0 \%)$ & $2(1.5 \%)$ & $0(0.0 \%)$ & $0(0.0 \%)$ & \\
\hline IIIA & $33(6.0 \%)$ & $8(5.8 \%)$ & $10(7.4 \%)$ & $4(2.7 \%)$ & $11(8.6 \%)$ & \\
\hline Postoperative pathology & & & & & & 0.009 \\
\hline Adenocarcinoma & $504(92.0 \%)$ & $122(87.8 \%)$ & $128(94.8 \%)$ & $132(90.4 \%)$ & $122(95.3 \%)$ & \\
\hline Squamous cell carcinoma & $37(6.8 \%)$ & $17(12.2 \%)$ & $4(3.0 \%)$ & $12(8.2 \%)$ & $4(3.1 \%)$ & \\
\hline Adenosquamous carcinoma & $7(1.3 \%)$ & $0(0.0 \%)$ & $3(2.2 \%)$ & $2(1.4 \%)$ & $2(1.6 \%)$ & \\
\hline Postoperative length of stay, days & $5.8 \pm 2.1$ & $5.7 \pm 1.7$ & $5.5 \pm 1.7$ & $6.3 \pm 2.8$ & $5.8 \pm 1.9$ & 0.093 \\
\hline Total hospital care costs (Renminbi) & $58351.3 \pm 9687.7$ & $60533.3 \pm 10223.6$ & $57968.9 \pm 8391.9$ & $58067.5 \pm 11048.8$ & $56708.7 \pm 8294.2$ & 0.006 \\
\hline
\end{tabular}

Values are presented as mean \pm standard deviation, $\mathrm{n}$ or $\mathrm{n}(\%)$. ASA, American Society of Anaesthesiologists; BMI, body mass index; FEV1, forced expiratory volume in $1 \mathrm{~s}$; FEV1\%, FEV1 as percentage of predicted; FVC, forced vital capacity; FVC\%, FVC as percentage of predicted; NSCLC, non-small cell lung cancer; PEF, peak expiratory flow; PEF\%, PEF as percentage of predicted. 
Table S2 Univariate results of postoperative outcomes based on PEF\%

\begin{tabular}{|c|c|c|c|c|c|c|}
\hline \multirow[b]{2}{*}{ Postoperative outcome } & \multicolumn{6}{|c|}{ PEF\% } \\
\hline & \multirow[t]{2}{*}{ Total $(n=548)$} & \multicolumn{4}{|c|}{$\begin{array}{l}\text { Quartile } 1(n=139) \text {, Quartile } 2(n=135) \text {, Quartile } 3(n=146) \text {, Quartile } 4(n=128) \text {, } \\
\quad \leq 49.0 \%>49.0-65.6 \% \quad>65.6-84.0 \% \quad>84.0 \%\end{array}$} & $P$ value \\
\hline \multicolumn{6}{|l|}{$\begin{array}{l}\text { Postoperative pulmonary } \\
\text { complications }\end{array}$} & \\
\hline $\begin{array}{l}\text { Acute respiratory distress } \\
\text { syndrome }\end{array}$ & $4(0.7 \%)$ & $1(0.7 \%)$ & $0(0.0 \%)$ & $2(1.4 \%)$ & $1(0.8 \%)$ & 0.451 \\
\hline Reintubation & $0(0.0 \%)$ & $0(0.0 \%)$ & $0(0.0 \%)$ & $0(0.0 \%)$ & $0(0.0 \%)$ & - \\
\hline Pulmonary embolism & $3(0.5 \%)$ & $1(0.7 \%)$ & $0(0.0 \%)$ & $2(1.4 \%)$ & $0(0.0 \%)$ & 0.237 \\
\hline Need for bedside bronchoscopy & $4(0.7 \%)$ & $0(0.0 \%)$ & $2(1.5 \%)$ & $1(0.7 \%)$ & $1(0.8 \%)$ & 0.414 \\
\hline Prolonged air leak & $10(1.8 \%)$ & $1(0.7 \%)$ & $3(2.2 \%)$ & $4(2.7 \%)$ & $2(1.6 \%)$ & 0.576 \\
\hline Failure to expand & $13(2.4 \%)$ & $5(3.6 \%)$ & $5(3.7 \%)$ & $1(0.7 \%)$ & $2(1.6 \%)$ & 0.207 \\
\hline Atelectasis & $13(2.4 \%)$ & $0(0.0 \%)$ & $6(4.4 \%)$ & $4(2.7 \%)$ & $3(2.3 \%)$ & 0.033 \\
\hline Pneumonia & 206 (37.6\%) & $64(46.0 \%)$ & $41(30.4 \%)$ & 49 (33.6\%) & $52(40.6 \%)$ & 0.033 \\
\hline Acute kidney injury & $12(2.2 \%)$ & $2(1.4 \%)$ & $4(3.0 \%)$ & $0(0.0 \%)$ & $6(4.7 \%)$ & 0.018 \\
\hline In-hospital mortality, n & $0(0.0 \%)$ & $0(0.0 \%)$ & $0(0.0 \%)$ & $0(0.0 \%)$ & $0(0.0 \%)$ & - \\
\hline Readmission within 30 days & $6(1.1 \%)$ & $2(1.4 \%)$ & $0(0.0 \%)$ & $3(2.1 \%)$ & $1(0.8 \%)$ & 0.238 \\
\hline
\end{tabular}

Values are presented as $\mathrm{n}(\%)$.

Table S3 Assignment of variables in multivariate analysis

\begin{tabular}{|c|c|}
\hline Variables & Assignment instruction \\
\hline ASA score & $A S A I=1, A S A \|=2$, ASA $\| I=3$ \\
\hline Age & $<60=1,60-=2$ \\
\hline Gender & Male $=1$, Female $=2$ \\
\hline BMI, $\mathrm{kg} / \mathrm{m}^{2}$ & $<24.0 \mathrm{~kg} / \mathrm{m}^{2}=1, \geq 24.0 \mathrm{~kg} / \mathrm{m}^{2}=2$ \\
\hline Smoking & $\mathrm{No}=0, \mathrm{Yes}=1$ \\
\hline Diabetes mellitus & $\mathrm{No}=0, \mathrm{Yes}=1$ \\
\hline Coronary heart disease & $\mathrm{No}=0, \mathrm{Yes}=1$ \\
\hline Intraoperative bleeding, mL & $<50 \mathrm{~mL}=1,50 \mathrm{~mL}-=2$ \\
\hline Length of operation, $\mathrm{h}$ & $<2 \mathrm{~h}=1,2 \mathrm{~h}-=2$ \\
\hline NSCLC staging & $I A=1, I B=2, \| A=3, I I B=4, I I I A=5$ \\
\hline Postoperative pathology & Adenocarcinoma =1; Squamous cell carcinoma $=2 ;$ Adenosquamous carcinoma $=3$ \\
\hline PEF\% & $\leq 49.0 \%=1,>49.0-65.6 \%=2,>65.6-84.0 \%=3,>84.0 \%=4$ \\
\hline FVC\% & $\leq 80.0 \%=1,>80.0-92.0 \%=2,>92.0-101.0 \%=3,>101.0 \%=4$ \\
\hline FEV1\% & $\leq 78.4 \%=1,>78.4-92.0 \%=2,>92.0-103.0 \%=3,>103.0 \%=4$ \\
\hline PEF, L/s & $\leq 3.3 \mathrm{~L} / \mathrm{s}=1,>3.3-4.4 \mathrm{~L} / \mathrm{s}=2,>4.4-5.8 \mathrm{~L} / \mathrm{s}=3,>5.8 \mathrm{~L} / \mathrm{s}=4$ \\
\hline FVC, L & $\leq 2.3 \mathrm{~L}=1,>2.3-2.9 \mathrm{~L}=2,>2.9-3.4 \mathrm{~L}=3,>3.4 \mathrm{~L}=4$ \\
\hline FEV1, L & $\leq 1.8 \mathrm{~L}=1,>1.8-2.3 \mathrm{~L}=2,>2.3-2.7 \mathrm{~L}=3,>2.7 \mathrm{~L}=4$ \\
\hline
\end{tabular}

ASA, American Society of Anaesthesiologists; BMI, body mass index; FEV1, forced expiratory volume in $1 \mathrm{~s}$; FEV1\%, FEV1 as percentage of predicted; FVC, forced vital capacity; FVC\%, FVC as percentage of predicted; NSCLC, non-small cell lung cancer; PEF, peak expiratory flow; PEF\%, PEF as percentage of predicted. 
Table S4 Clinical characteristics of patients based on FVC\%

\begin{tabular}{|c|c|c|c|c|c|}
\hline \multirow[b]{2}{*}{ Characteristic } & \multicolumn{5}{|c|}{ FVC $\%$} \\
\hline & $\begin{array}{l}\text { Quartile } 1(n=145) \\
\quad \leq 80.0 \%\end{array}$ & $\begin{array}{c}\text { Quartile } 2(n=136) \\
\quad>80.0-92.0 \%\end{array}$ & $\begin{array}{c}\text { Quartile } 3(n=133) \\
>92.0-101.0 \%\end{array}$ & $\begin{array}{c}\text { Quartile } 4(n=134), \\
>101.0 \%\end{array}$ & $P$ value \\
\hline ASA score & & & & & 0.343 \\
\hline I & 115 (79.3\%) & $113(83.1 \%)$ & $113(85.0 \%)$ & $118(88.1 \%)$ & \\
\hline II & $20(13.8 \%)$ & $18(13.2 \%)$ & $17(12.8 \%)$ & $11(8.2 \%)$ & \\
\hline III & $10(6.9 \%)$ & $5(3.7 \%)$ & $3(2.3 \%)$ & $5(3.7 \%)$ & \\
\hline Age & $66.7 \pm 9.5$ & $63.2 \pm 10.8$ & $61.2 \pm 10.5$ & $60.4 \pm 10.5$ & 0.000 \\
\hline Gender (female/male) & $81 / 64$ & $96 / 40$ & $74 / 59$ & $80 / 54$ & 0.038 \\
\hline Weight, kg & $64.6 \pm 9.7$ & $60.7 \pm 10.3$ & $61.0 \pm 8.8$ & $58.9 \pm 9.5$ & 0.000 \\
\hline BMI, $\mathrm{kg} / \mathrm{m}^{2}$ & $24.2 \pm 2.9$ & $23.1 \pm 2.9$ & $22.9 \pm 2.4$ & $22.4 \pm 2.8$ & 0.000 \\
\hline Smoking & $45(31.0 \%)$ & $28(20.6 \%)$ & $36(27.1 \%)$ & $37(27.6 \%)$ & 0.255 \\
\hline Diabetes mellitus & $17(11.7 \%)$ & $10(7.4 \%)$ & $11(8.3 \%)$ & $10(7.5 \%)$ & 0.522 \\
\hline Coronary heart disease & $6(4.1 \%)$ & $0(0.0 \%)$ & $1(0.8 \%)$ & $2(1.5 \%)$ & 0.026 \\
\hline FEV1, L & $1.9 \pm 0.4$ & $2.2 \pm 0.5$ & $2.5 \pm 0.5$ & $2.8 \pm 0.5$ & 0.000 \\
\hline FVC, L & $2.3 \pm 0.5$ & $2.7 \pm 0.6$ & $3.2 \pm 0.6$ & $3.5 \pm 0.7$ & 0.000 \\
\hline PEF, L/s & $3.9 \pm 1.5$ & $4.1 \pm 1.6$ & $5.0 \pm 1.8$ & $5.8 \pm 1.9$ & 0.000 \\
\hline FEV1\% & $(73.8 \pm 10.9) \%$ & $(86.7 \pm 10.2) \%$ & $(95.9 \pm 10.6) \%$ & $(109.3 \pm 13.1) \%$ & 0.000 \\
\hline FVC\% & $(72.0 \pm 6.4) \%$ & $(86.7 \pm 3.2) \%$ & $(96.5 \pm 2.7) \%$ & $(111.6 \pm 9.0) \%$ & 0.000 \\
\hline PEF\% & $(55.9 \pm 20.0) \%$ & $(62.1 \pm 20.9) \%$ & $(71.4 \pm 24.0) \%$ & $(82.2 \pm 22.5) \%$ & 0.000 \\
\hline Intraoperative bleeding, mL & $53.8 \pm 45.1$ & $45.7 \pm 23.8$ & $48.0 \pm 25.6$ & $40.8 \pm 26.1$ & 0.021 \\
\hline Intraoperative blood transfusion, $\mathrm{mL}$ & 0 & 0 & 0 & 0 & - \\
\hline Length of operation, min & $136.0 \pm 42.8$ & $135.0 \pm 34.8$ & $127.6 \pm 30.0$ & $131.4 \pm 32.6$ & 0.259 \\
\hline NSCLC staging & & & & & 0.003 \\
\hline IA & $124(85.5 \%)$ & $105(77.2 \%)$ & $111(83.5 \%)$ & 107 (79.9\%) & \\
\hline IB & $15(10.3 \%)$ & $12(8.8 \%)$ & $6(4.5 \%)$ & $4(3.0 \%)$ & \\
\hline$\| \mathrm{A}$ & $4(2.8 \%)$ & $7(5.1 \%)$ & $7(5.3 \%)$ & $11(8.2 \%)$ & \\
\hline IIB & $1(0.7 \%)$ & $0(0.0 \%)$ & $1(0.8 \%)$ & $0(0.0 \%)$ & \\
\hline IIIA & $1(0.7 \%)$ & $12(8.8 \%)$ & $8(6.0 \%)$ & $12(9.0 \%)$ & \\
\hline Postoperative pathology & & & & & 0.577 \\
\hline Adenocarcinoma, $\mathrm{n}$ & $130(89.7 \%)$ & $125(91.9 \%)$ & $122(91.7 \%)$ & 127 (94.8\%) & \\
\hline Squamous cell carcinoma, $\mathrm{n}$ & $14(9.7 \%)$ & $8(5.9 \%)$ & $9(6.8 \%)$ & $6(4.5 \%)$ & \\
\hline Adenosquamous carcinoma, $\mathrm{n}$ & $1(0.7 \%)$ & $3(2.2 \%)$ & $2(1.5 \%)$ & $1(0.7 \%)$ & \\
\hline Postoperative length of stay, days & $5.9 \pm 2.4$ & $5.7 \pm 1.9$ & $5.8 \pm 2.2$ & $5.9 \pm 2.0$ & 0.735 \\
\hline Total hospital care costs (Renminbi) & $59940.6 \pm 10768.4$ & $58222.5 \pm 9788.9$ & $58621.0 \pm 9679.4$ & $56494.4 \pm 7992.4$ & 0.120 \\
\hline
\end{tabular}

Values are presented as mean \pm standard deviation, $\mathrm{n}$ or $\mathrm{n}(\%)$. ASA, American Society of Anaesthesiologists; BMI, body mass index; FEV1, forced expiratory volume in $1 \mathrm{~s}$; FEV1\%, FEV1 as percentage of predicted; FVC, forced vital capacity; FVC\%, FVC as percentage of predicted; NSCLC, non-small cell lung cancer; PEF, peak expiratory flow; PEF\%, PEF as percentage of predicted. 
Table S5 Univariate results of postoperative outcomes based on FVC\%

\begin{tabular}{|c|c|c|c|c|c|}
\hline \multirow[b]{2}{*}{ Postoperative outcome } & \multicolumn{5}{|c|}{ FVC \% } \\
\hline & $\begin{array}{l}\text { Quartile } 1(n=145) \\
\quad \leq 80.0 \%\end{array}$ & $\begin{array}{l}\text { Quartile } 2(n=136) \\
\quad>80.0-92.0 \%\end{array}$ & $\begin{array}{l}\text { Quartile } 3(n=133) \\
\quad>92.0-101.0 \%\end{array}$ & $\begin{array}{c}\text { Quartile } 4(n=134) \\
>101.0 \%\end{array}$ & $P$ value \\
\hline \multicolumn{6}{|l|}{ Postoperative pulmonary complications } \\
\hline Acute respiratory distress syndrome & $2(1.4 \%)$ & $0(0.0 \%)$ & $1(0.8 \%)$ & $1(0.7 \%)$ & 0.447 \\
\hline Reintubation & $0(0.0 \%)$ & $0(0.0 \%)$ & $0(0.0 \%)$ & $0(0.0 \%)$ & - \\
\hline Pulmonary embolism & $2(1.4 \%)$ & $0(0.0 \%)$ & $1(0.8 \%)$ & $0(0.0 \%)$ & 0.226 \\
\hline Need for bedside bronchoscopy & $0(0.0 \%)$ & $2(1.5 \%)$ & $2(1.5 \%)$ & $0(0.0 \%)$ & 0.126 \\
\hline Prolonged air leak & $2(1.4 \%)$ & $1(0.7 \%)$ & $3(2.3 \%)$ & $4(3.0 \%)$ & 0.510 \\
\hline Failure to expand & $2(1.4 \%)$ & $6(4.4 \%)$ & $0(0.0 \%)$ & $5(3.7 \%)$ & 0.019 \\
\hline Atelectasis & $4(2.8 \%)$ & $4(2.9 \%)$ & $2(1.5 \%)$ & $3(2.2 \%)$ & 0.856 \\
\hline Pneumonia & $64(44.1 \%)$ & $62(45.6 \%)$ & $36(27.1 \%)$ & $44(32.8 \%)$ & 0.003 \\
\hline Acute kidney injury & $4(2.8 \%)$ & $3(2.2 \%)$ & $0(0.0 \%)$ & $5(3.7 \%)$ & 0.062 \\
\hline In-hospital mortality, n & $0(0.0 \%)$ & $0(0.0 \%)$ & $0(0.0 \%)$ & $0(0.0 \%)$ & - \\
\hline Readmission within 30 days & $1(0.7 \%)$ & $0(0.0 \%)$ & $2(1.5 \%)$ & $3(2.2 \%)$ & 0.197 \\
\hline
\end{tabular}

Values are presented as $n(\%)$. 
Table S6 Clinical characteristics of patients based on FEV1\%

\begin{tabular}{|c|c|c|c|c|c|}
\hline \multirow[b]{2}{*}{ Characteristic } & \multicolumn{5}{|c|}{ FEV1\% } \\
\hline & $\begin{array}{l}\text { Quartile } 1(n=138) \\
\quad \leq 78.4 \%\end{array}$ & $\begin{array}{l}\text { Quartile } 2(n=148) \\
\quad>78.4-92.0 \%\end{array}$ & $\begin{array}{c}\text { Quartile } 3(n=130) \\
>92.0-103.0 \%\end{array}$ & $\begin{array}{c}\text { Quartile } 4(n=132), \\
\quad>103.0 \%\end{array}$ & $P$ value \\
\hline ASA score & & & & & 0.228 \\
\hline I & $111(80.4 \%)$ & $125(84.5 \%)$ & $107(82.3 \%)$ & 116 (87.9\%) & \\
\hline II & $16(11.6 \%)$ & $19(12.8 \%)$ & $18(13.8 \%)$ & $13(9.8 \%)$ & \\
\hline III & $11(8.0 \%)$ & $4(2.7 \%)$ & $5(3.8 \%)$ & $3(2.3 \%)$ & \\
\hline Age & $66.0 \pm 9.3$ & $63.1 \pm 10.9$ & $61.3 \pm 11.4$ & $61.3 \pm 10.0$ & 0.000 \\
\hline Gender (female/male) & $81 / 57$ & $92 / 56$ & $71 / 59$ & $87 / 45$ & 0.278 \\
\hline Weight, kg & $63.6 \pm 11.1$ & $61.7 \pm 8.8$ & $61.1 \pm 10.2$ & $59.0 \pm 8.5$ & 0.004 \\
\hline BMI, $\mathrm{kg} / \mathrm{m}^{2}$ & $23.8 \pm 3.1$ & $23.1 \pm 2.8$ & $22.9 \pm 2.7$ & $22.7 \pm 2.6$ & 0.006 \\
\hline Smoking & $49(35.5 \%)$ & $39(26.4 \%)$ & $34(26.2 \%)$ & $24(18.2 \%)$ & 0.015 \\
\hline Diabetes mellitus & $16(11.6 \%)$ & $10(6.8 \%)$ & $11(8.5 \%)$ & $11(8.3 \%)$ & 0.537 \\
\hline Coronary heart disease & $6(4.3 \%)$ & $0(0.0 \%)$ & $3(2.3 \%)$ & $0(0.0 \%)$ & 0.003 \\
\hline FEV1, L & $1.8 \pm 0.4$ & $2.2 \pm 0.4$ & $2.6 \pm 0.5$ & $2.8 \pm 0.5$ & 0.000 \\
\hline FVC, L & $2.5 \pm 0.6$ & $2.8 \pm 0.7$ & $3.2 \pm 0.7$ & $3.3 \pm 0.7$ & 0.000 \\
\hline PEF, L/s & $3.3 \pm 1.3$ & $4.2 \pm 1.6$ & $5.4 \pm 1.7$ & $5.9 \pm 1.8$ & 0.000 \\
\hline FEV1\% & $(69.0 \pm 7.4) \%$ & $(86.1 \pm 4.1) \%$ & $(97.7 \pm 3.2) \%$ & $(113.1 \pm 9.0) \%$ & 0.000 \\
\hline FVC\% & $(76.0 \pm 11.9) \%$ & $(86.7 \pm 9.4) \%$ & $(96.3 \pm 9.7) \%$ & $(107.5 \pm 11.4) \%$ & 0.000 \\
\hline PEF\% & $(47.9 \pm 14.9) \%$ & $(62.2 \pm 19.8) \%$ & $(76.6 \pm 20.6) \%$ & $(85.4 \pm 21.4) \%$ & 0.000 \\
\hline Intraoperative bleeding, mL & $51.0 \pm 42.8$ & $49.3 \pm 32.5$ & $45.4 \pm 21.5$ & $42.7 \pm 25.7$ & 0.475 \\
\hline Intraoperative blood transfusion, $\mathrm{mL}$ & 0 & 0 & 0 & 0 & - \\
\hline Length of operation, min & $133.4 \pm 40.7$ & $133.2 \pm 35.2$ & $134.9 \pm 33.3$ & $128.9 \pm 32.5$ & 0.613 \\
\hline NSCLC staging & & & & & 0.030 \\
\hline IA & $107(77.5 \%)$ & $127(85.8 \%)$ & $105(80.8 \%)$ & $108(81.8 \%)$ & \\
\hline IB & $18(13.0 \%)$ & $8(5.4 \%)$ & $8(6.2 \%)$ & $3(2.3 \%)$ & \\
\hline$\| \mathrm{A}$ & $6(4.3 \%)$ & $4(2.7 \%)$ & $11(8.5 \%)$ & $8(6.1 \%)$ & \\
\hline IIB & $0(0.0 \%)$ & $1(0.7 \%)$ & $0(0.0 \%)$ & $1(0.8 \%)$ & \\
\hline IIIA & $7(5.1 \%)$ & $8(5.4 \%)$ & $6(4.6 \%)$ & $12(9.1 \%)$ & \\
\hline Postoperative pathology & & & & & 0.034 \\
\hline Adenocarcinoma, $\mathrm{n}$ & $121(87.7 \%)$ & $138(93.2 \%)$ & $117(90.0 \%)$ & $128(97.0 \%)$ & \\
\hline Squamous cell carcinoma, $\mathrm{n}$ & $16(11.6 \%)$ & $9(6.1 \%)$ & $9(6.9 \%)$ & $3(2.3 \%)$ & \\
\hline Adenosquamous carcinoma, $\mathrm{n}$ & $1(0.7 \%)$ & $1(0.7 \%)$ & $4(3.1 \%)$ & $1(0.8 \%)$ & \\
\hline Postoperative length of stay, days & $5.6 \pm 1.6$ & $6.0 \pm 2.6$ & $5.9 \pm 2.2$ & $5.9 \pm 2.0$ & 0.394 \\
\hline Total hospital care costs (Renminbi) & $59089.3 \pm 9764.3$ & $59150.7 \pm 10326.5$ & $59378.5 \pm 9167.6$ & $55671.8 \pm 8954.3$ & 0.004 \\
\hline
\end{tabular}

Values are presented as mean \pm standard deviation, $\mathrm{n}$ or $\mathrm{n}(\%)$. ASA, American Society of Anaesthesiologists; BMI, body mass index; FEV1, forced expiratory volume in $1 \mathrm{~s}$; FEV1\%, FEV1 as percentage of predicted; FVC, forced vital capacity; FVC\%, FVC as percentage of predicted; NSCLC, non-small cell lung cancer; PEF, peak expiratory flow; PEF\%, PEF as percentage of predicted. 
Table S7 Univariate results of postoperative outcomes based on FEV1\%

\begin{tabular}{|c|c|c|c|c|c|}
\hline \multirow[b]{2}{*}{ Postoperative outcome } & \multicolumn{5}{|c|}{ FEV1\% } \\
\hline & $\begin{array}{l}\text { Quartile } 1(n=138) \\
\quad \leq 78.4 \%\end{array}$ & $\begin{array}{c}\text { Quartile } 2(\mathrm{n}=148) \\
\quad>78.4-92.0 \%\end{array}$ & $\begin{array}{c}\text { Quartile } 3(n=130) \\
>92.0-103.0 \%\end{array}$ & $\begin{array}{l}\text { Quartile } 4(n=132) \\
\quad>103.0 \%\end{array}$ & $P$ value \\
\hline \multicolumn{6}{|l|}{ Postoperative pulmonary complications } \\
\hline Acute respiratory distress syndrome & $0(0.0 \%)$ & $2(1.4 \%)$ & $1(0.8 \%)$ & $1(0.8 \%)$ & 0.448 \\
\hline Reintubation & $0(0.0 \%)$ & $0(0.0 \%)$ & $0(0.0 \%)$ & $0(0.0 \%)$ & - \\
\hline Pulmonary embolism & $0(0.0 \%)$ & $2(1.4 \%)$ & $1(0.8 \%)$ & $0(0.0 \%)$ & 0.230 \\
\hline Need for bedside bronchoscopy & $0(0.0 \%)$ & $2(1.4 \%)$ & $2(1.5 \%)$ & $0(0.0 \%)$ & 0.140 \\
\hline Prolonged air leak & $0(0.0 \%)$ & $6(4.1 \%)$ & $2(1.5 \%)$ & $2(1.5 \%)$ & 0.041 \\
\hline Failure to expand & $4(2.9 \%)$ & $4(2.7 \%)$ & $4(3.1 \%)$ & $1(0.8 \%)$ & 0.477 \\
\hline Atelectasis & $2(1.4 \%)$ & $3(2.0 \%)$ & $3(2.3 \%)$ & $5(3.8 \%)$ & 0.650 \\
\hline Pneumonia & $56(40.6 \%)$ & $64(43.2 \%)$ & $43(33.1 \%)$ & $43(32.6 \%)$ & 0.166 \\
\hline Acute kidney injury & $2(1.4 \%)$ & $6(4.1 \%)$ & $0(0.0 \%)$ & $4(3.0 \%)$ & 0.037 \\
\hline In-hospital mortality, n & $0(0.0 \%)$ & $0(0.0 \%)$ & $0(0.0 \%)$ & $0(0.0 \%)$ & - \\
\hline Readmission within 30 days & $0(0.0 \%)$ & $1(0.7 \%)$ & $2(1.5 \%)$ & $3(2.3 \%)$ & 0.186 \\
\hline
\end{tabular}

Values are presented as $n(\%)$. 
Table S8 Full-model multivariate results of 4 groups of different PEF\%

\begin{tabular}{|c|c|c|c|}
\hline \multirow{2}{*}{ Variables } & \multicolumn{3}{|c|}{ Postoperative pneumonia } \\
\hline & OR & $95 \% \mathrm{Cl}$ & $P$ value \\
\hline ASA score & & & 0.022 \\
\hline I & 1.000 & & \\
\hline II & 3.124 & $1.325-7.366$ & 0.009 \\
\hline III & 2.813 & $0.730-10.847$ & 0.133 \\
\hline \multicolumn{4}{|l|}{ Age } \\
\hline$<60$ & 1.000 & & \\
\hline $60-$ & 1.527 & $1.012-2.303$ & 0.044 \\
\hline \multicolumn{4}{|l|}{ Gender } \\
\hline Male & 1.000 & & \\
\hline Female & 1.322 & $0.750-2.332$ & 0.335 \\
\hline \multicolumn{4}{|l|}{ BMI } \\
\hline$<24.0 \mathrm{~kg} / \mathrm{m}^{2}$ & 1.000 & & \\
\hline$\geq 24.0 \mathrm{~kg} / \mathrm{m}^{2}$ & 1.642 & $1.103-2.444$ & 0.015 \\
\hline \multicolumn{4}{|l|}{ Smoking } \\
\hline No & 1.000 & & \\
\hline Yes & 0.912 & $0.471-1.765$ & 0.784 \\
\hline \multicolumn{4}{|l|}{ Diabetes mellitus } \\
\hline No & 1.000 & & \\
\hline Yes & 0.101 & $0.035-0.292$ & 0.000 \\
\hline \multicolumn{4}{|l|}{ Coronary heart disease } \\
\hline No & 1.000 & & \\
\hline Yes & 0.468 & $0.069-3.188$ & 0.438 \\
\hline \multicolumn{4}{|l|}{ Intraoperative bleeding } \\
\hline$<50 \mathrm{~mL}$ & 1.000 & & \\
\hline $50 \mathrm{~mL}-$ & 0.967 & $0.647-1.446$ & 0.870 \\
\hline \multicolumn{4}{|l|}{ Length of operation } \\
\hline$<2 \mathrm{~h}$ & 1.000 & & \\
\hline $2 \mathrm{~h}-$ & 1.547 & $1.030-2.326$ & 0.036 \\
\hline NSCLC staging & & & 0.479 \\
\hline IA & 1.000 & & \\
\hline IB & 0.989 & $0.461-2.124$ & 0.978 \\
\hline$\| \mathrm{A}$ & 2.077 & $0.895-4.821$ & 0.089 \\
\hline IIB & 3.117 & $0.179-54.389$ & 0.436 \\
\hline IIIA & 1.014 & $0.460-2.239$ & 0.972 \\
\hline Postoperative pathology & & & 0.223 \\
\hline Adenocarcinoma & 1.000 & & \\
\hline Squamous cell carcinoma & 2.052 & $0.910-4.627$ & 0.083 \\
\hline Adenosquamous carcinoma & 0.000 & 0.000 & 0.999 \\
\hline PEF\% & & & 0.013 \\
\hline$\leq 49.0 \%$ & 2.076 & $1.211-3.558$ & 0.008 \\
\hline$>49.0-65.6 \%$ & 1.000 & & \\
\hline$>65.6-84.0 \%$ & 1.157 & $0.677-1.978$ & 0.595 \\
\hline$>84.0 \%$ & 1.962 & $1.129-3.411$ & 0.017 \\
\hline
\end{tabular}

Results of binary logistics regression are presented as adjusted OR, 95\% Cl and P value. ASA, American Society of Anaesthesiologists BMI, body mass index; Cl, confidence interval; NSCLC, non-small cell lung cancer; OR, odds ratio; PEF\%, peak expiratory flow as a percentage of predicted. 
Table S9 Full-model multivariate results of 4 groups of different FVC\%

\begin{tabular}{|c|c|c|c|}
\hline \multirow{2}{*}{ Variables } & \multicolumn{3}{|c|}{ Postoperative pneumonia } \\
\hline & OR & $95 \% \mathrm{Cl}$ & $P$ value \\
\hline ASA score & & & 0.021 \\
\hline I & 1.000 & & \\
\hline II & 3.188 & $1.339-7.587$ & 0.009 \\
\hline III & 2.821 & $0.740-10.757$ & 0.129 \\
\hline \multicolumn{4}{|l|}{ Age } \\
\hline$<60$ & 1.000 & & \\
\hline $60-$ & 1.297 & $0.862-1.952$ & 0.213 \\
\hline \multicolumn{4}{|l|}{ Gender } \\
\hline Male & 1.000 & & \\
\hline Female & 1.209 & $0.686-2.131$ & 0.512 \\
\hline \multicolumn{4}{|l|}{ BMI } \\
\hline$<24.0 \mathrm{~kg} / \mathrm{m}^{2}$ & 1.000 & & \\
\hline$\geq 24.0 \mathrm{~kg} / \mathrm{m}^{2}$ & 1.461 & $0.977-2.185$ & 0.065 \\
\hline \multicolumn{4}{|l|}{ Smoking } \\
\hline No & 1.000 & & \\
\hline Yes & 0.937 & $0.487-1.801$ & 0.844 \\
\hline \multicolumn{4}{|l|}{ Diabetes mellitus } \\
\hline No & 1.000 & & \\
\hline Yes & 0.108 & $0.037-0.315$ & 0.000 \\
\hline \multicolumn{4}{|l|}{ Coronary heart disease } \\
\hline No & 1.000 & & \\
\hline Yes & 0.470 & $0.069-3.187$ & 0.439 \\
\hline \multicolumn{4}{|l|}{ Intraoperative bleeding } \\
\hline$<50 \mathrm{~mL}$ & 1.000 & & \\
\hline $50 \mathrm{~mL}-$ & 0.982 & $0.654-1.474$ & 0.930 \\
\hline \multicolumn{4}{|l|}{ Length of operation } \\
\hline$<2 \mathrm{~h}$ & 1.000 & & \\
\hline $2 \mathrm{~h}-$ & 1.514 & $1.009-2.270$ & 0.045 \\
\hline NSCLC staging & & & 0.385 \\
\hline IA & 1.000 & & \\
\hline IB & 0.782 & $0.363-1.686$ & 0.530 \\
\hline$\| \mathrm{A}$ & 2.237 & $0.946-5.289$ & 0.067 \\
\hline IIB & 2.043 & $0.116-35.835$ & 0.625 \\
\hline IIIA & 1.145 & $0.514-2.550$ & 0.741 \\
\hline Postoperative pathology & & & 0.157 \\
\hline Adenocarcinoma & 1.000 & & \\
\hline Squamous cell carcinoma & 2.208 & $0.985-4.949$ & 0.054 \\
\hline Adenosquamous carcinoma & 0.000 & 0.000 & 0.999 \\
\hline FVC \% & & & 0.012 \\
\hline$\leq 80.0 \%$ & 2.125 & $1.226-3.683$ & 0.007 \\
\hline$>80.0-92.0 \%$ & 2.230 & $1.298-3.832$ & 0.004 \\
\hline$>92.0-101.0 \%$ & 1.000 & & \\
\hline$>101.0 \%$ & 1.399 & $0.802-2.440$ & 0.237 \\
\hline
\end{tabular}

Results of binary logistics regression are presented as adjusted OR, $95 \% \mathrm{Cl}$ and $\mathrm{P}$ value. ASA, American Society of Anaesthesiologists BMI, body mass index; Cl, confidence interval; FVC\%, forced vital capacity as percentage of predicted; NSCLC, non-small cell lung cancer; OR, odds ratio. 
Table S10 Full-model multivariate results of 4 groups of different FEV1\%

\begin{tabular}{|c|c|c|c|}
\hline \multirow{2}{*}{ Variables } & \multicolumn{3}{|c|}{ Postoperative pneumonia } \\
\hline & OR & $95 \% \mathrm{Cl}$ & $P$ value \\
\hline ASA score & & & 0.022 \\
\hline I & 1.000 & & \\
\hline II & 3.121 & $1.325-7.354$ & 0.009 \\
\hline III & 2.813 & $0.745-10.623$ & 0.127 \\
\hline \multicolumn{4}{|l|}{ Age } \\
\hline$<60$ & 1.000 & & \\
\hline $60-$ & 1.344 & $0.898-2.012$ & 0.151 \\
\hline \multicolumn{4}{|l|}{ Gender } \\
\hline Male & 1.000 & & \\
\hline Female & 1.266 & $0.716-2.239$ & 0.417 \\
\hline \multicolumn{4}{|l|}{ BMI } \\
\hline$<24.0 \mathrm{~kg} / \mathrm{m}^{2}$ & 1.000 & & \\
\hline$\geq 24.0 \mathrm{~kg} / \mathrm{m}^{2}$ & 1.532 & $1.029-2.282$ & 0.036 \\
\hline \multicolumn{4}{|l|}{ Smoking } \\
\hline No & 1.000 & & \\
\hline Yes & 0.932 & $0.482-1.801$ & 0.834 \\
\hline \multicolumn{4}{|l|}{ Diabetes mellitus } \\
\hline No & 1.000 & & \\
\hline Yes & 0.114 & $0.040-0.330$ & 0.000 \\
\hline \multicolumn{4}{|l|}{ Coronary heart disease } \\
\hline No & 1.000 & & \\
\hline Yes & 0.500 & $0.074-3.363$ & 0.476 \\
\hline \multicolumn{4}{|l|}{ Intraoperative bleeding } \\
\hline$<50 \mathrm{~mL}$ & 1.000 & & \\
\hline $50 \mathrm{~mL}-$ & 0.983 & $0.659-1.466$ & 0.934 \\
\hline \multicolumn{4}{|l|}{ Length of operation } \\
\hline$<2 \mathrm{~h}$ & 1.000 & & \\
\hline $2 \mathrm{~h}-$ & 1.522 & $1.016-2.279$ & 0.042 \\
\hline NSCLC staging & & & 0.457 \\
\hline IA & 1.000 & & \\
\hline IB & 0.826 & $0.382-1.786$ & 0.627 \\
\hline$\| \mathrm{A}$ & 2.145 & $0.920-5.001$ & 0.077 \\
\hline IIB & 1.833 & $0.107-31.433$ & 0.676 \\
\hline IIIA & 1.120 & $0.513-2.441$ & 0.776 \\
\hline Postoperative pathology & & & 0.151 \\
\hline Adenocarcinoma & 1.000 & & \\
\hline Squamous cell carcinoma & 2.214 & $0.994-4.933$ & 0.052 \\
\hline Adenosquamous carcinoma & 0.000 & 0.000 & 0.999 \\
\hline FEV1\% & & & 0.349 \\
\hline$\leq 78.4 \%$ & 1.309 & $0.755-2.269$ & 0.338 \\
\hline$>78.4-92.0 \%$ & 1.465 & $0.876-2.448$ & 0.146 \\
\hline$>92.0-103.0 \%$ & 0.979 & $0.564-1.700$ & 0.940 \\
\hline$>103.0 \%$ & 1.000 & & \\
\hline
\end{tabular}

Results of binary logistics regression are presented as adjusted OR, 95\% Cl and P value. ASA, American Society of Anaesthesiologists; $\mathrm{BMI}$, body mass index; $\mathrm{Cl}$, confidence interval; FEV1\%, forced expiratory volume in $1 \mathrm{~s}$ as percentage of predicted; NSCLC, non-small cell lung cancer; OR, odds ratio. 
Table S11 Mortality following video-assisted thoracic surgery lobectomy for lung cancer based on FVC\%

\begin{tabular}{|c|c|c|c|c|c|}
\hline \multirow[b]{2}{*}{ Variables } & \multicolumn{5}{|c|}{ FVC \% } \\
\hline & $\begin{array}{l}\text { Quartile } 1(n=145) \\
\quad \leq 80.0 \%\end{array}$ & $\begin{array}{c}\text { Quartile } 2(n=136), \\
\quad>80.0-92.0 \%\end{array}$ & $\begin{array}{c}\text { Quartile } 3(n=133) \\
>92.0-101.0 \%\end{array}$ & $\begin{array}{c}\text { Quartile } 4(n=134) \text {, } \\
>101.0 \%\end{array}$ & $P$ value \\
\hline Number of deaths & $1(0.7 \%)$ & $3(2.2 \%)$ & $1(0.8 \%)$ & $4(3.0 \%)$ & 0.351 \\
\hline Cause of death & & & & & 0.256 \\
\hline Postoperative complication & $0(0.0 \%)$ & $0(0.0 \%)$ & $0(0.0 \%)$ & $0(0.0 \%)$ & \\
\hline Cancer related & $0(0.0 \%)$ & $2(66.7 \%)$ & $0(0.0 \%)$ & $3(75.0 \%)$ & \\
\hline Non-cancer related & $0(0.0 \%)$ & $0(0.0 \%)$ & $0(0.0 \%)$ & $0(0.0 \%)$ & \\
\hline Uncertain & $1(100.0 \%)$ & $1(33.3 \%)$ & $1(100.0 \%)$ & $1(25.0 \%)$ & \\
\hline
\end{tabular}

Values are presented as $\mathrm{n}(\%)$. FVC\%, forced vital capacity as percentage of predicted.

Table S12 Mortality following video-assisted thoracic surgery lobectomy for lung cancer based on FEV1\%

\begin{tabular}{|c|c|c|c|c|c|}
\hline \multirow[b]{2}{*}{ Variables } & \multicolumn{5}{|c|}{ FEV1\% } \\
\hline & $\begin{array}{l}\text { Quartile } 1(n=138) \\
\quad \leq 78.4 \%\end{array}$ & $\begin{array}{c}\text { Quartile } 2(n=148) \\
>78.4-92.0 \%\end{array}$ & $\begin{array}{c}\text { Quartile } 3(n=130) \\
>92.0-103.0 \%\end{array}$ & $\begin{array}{c}\text { Quartile } 4(n=132) \text {, } \\
\quad>103.0 \%\end{array}$ & $P$ value \\
\hline Number of deaths & $2(1.5 \%)$ & $1(0.7 \%)$ & $2(1.5 \%)$ & $4(3.0 \%)$ & 0.498 \\
\hline Cause of death & & & & & 0.022 \\
\hline Postoperative complication & $0(0.0 \%)$ & $0(0.0 \%)$ & $0(0.0 \%)$ & $0(0.0 \%)$ & \\
\hline Cancer related & $1(50.0 \%)$ & $0(0.0 \%)$ & $0(0.0 \%)$ & $4(100.0 \%)$ & \\
\hline Non-cancer related & $0(0.0 \%)$ & $0(0.0 \%)$ & $0(0.0 \%)$ & $0(0.0 \%)$ & \\
\hline Uncertain & $1(50.0 \%)$ & $1(100.0 \%)$ & $2(100.0 \%)$ & $0(0.0 \%)$ & \\
\hline
\end{tabular}

Values are presented as $\mathrm{n}(\%)$. FEV1\%, forced expiratory volume in $1 \mathrm{~s}$ as percentage of predicted. 
Table S13 Multivariate Cox regression results for overall survival based on PEF\%

\begin{tabular}{|c|c|c|c|}
\hline Variables & $\mathrm{HR}$ & $95 \% \mathrm{Cl}$ & $P$ value \\
\hline ASA score & & & 0.999 \\
\hline I & 1.000 & & \\
\hline ॥ & 0.000 & 0.000 & 0.963 \\
\hline III & 0.000 & 0.000 & 0.972 \\
\hline \multicolumn{4}{|l|}{ Age } \\
\hline$<60$ & 1.000 & & \\
\hline $60-$ & 0.772 & $0.184-3.241$ & 0.724 \\
\hline \multicolumn{4}{|l|}{ Gender } \\
\hline Male & 1.000 & & \\
\hline Female & 0.648 & $0.062-6.740$ & 0.717 \\
\hline \multicolumn{4}{|l|}{ BMI } \\
\hline$<24.0 \mathrm{~kg} / \mathrm{m}^{2}$ & 1.000 & & \\
\hline$\geq 24.0 \mathrm{~kg} / \mathrm{m}^{2}$ & 0.695 & $0.131-3.695$ & 0.669 \\
\hline \multicolumn{4}{|l|}{ Smoking } \\
\hline No & 1.000 & & \\
\hline Yes & 5.083 & $0.616-41.916$ & 0.131 \\
\hline \multicolumn{4}{|l|}{ Diabetes mellitus } \\
\hline No & 1.000 & & \\
\hline Yes & 0.000 & 0.000 & 0.959 \\
\hline \multicolumn{4}{|l|}{ Coronary heart disease } \\
\hline No & 1.000 & & \\
\hline Yes & 0.000 & 0.000 & 0.996 \\
\hline \multicolumn{4}{|l|}{ Intraoperative bleeding } \\
\hline$<50 \mathrm{~mL}$ & 1.000 & & \\
\hline $50 \mathrm{~mL}-$ & 6.692 & $0.632-70.866$ & 0.114 \\
\hline \multicolumn{4}{|l|}{ Length of operation } \\
\hline$<2 \mathrm{~h}$ & 1.000 & & \\
\hline $2 \mathrm{~h}-$ & 1.620 & $0.300-8.755$ & 0.575 \\
\hline NSCLC staging & & & 0.121 \\
\hline IA & 1.000 & & \\
\hline IB & 0.000 & 0.000 & 0.969 \\
\hline IIA & 0.000 & 0.000 & 0.971 \\
\hline IIB & 0.000 & 0.000 & 0.998 \\
\hline IIIA & 13.667 & $2.047-91.264$ & 0.007 \\
\hline Postoperative pathology & & & 0.999 \\
\hline Adenocarcinoma & 1.000 & & \\
\hline Squamous cell carcinoma & 0.000 & 0.000 & 0.960 \\
\hline Adenosquamous carcinoma & 0.000 & 0.000 & 0.985 \\
\hline PEF\% & & & 0.533 \\
\hline$\leq 49.0 \%$ & 0.737 & $0.107-5.076$ & 0.757 \\
\hline$>49.0-65.6 \%$ & 0.279 & $0.029-2.678$ & 0.269 \\
\hline$>65.6-84.0 \%$ & 0.263 & $0.028-2.510$ & 0.246 \\
\hline$>84.0 \%$ & 1.000 & & \\
\hline
\end{tabular}

Results of binary logistics regression are presented as adjusted $\mathrm{HR}, 95 \% \mathrm{Cl}$ and $\mathrm{P}$ value. ASA, American Society of Anaesthesiologists: $\mathrm{BMI}$, body mass index; $\mathrm{Cl}$, confidence interval; HR, hazard ratio; NSCLC, non-small cell lung cancer; PEF\%, peak expiratory flow as a percentage of predicted. 
Table S14 Multivariate Cox regression results for overall survival based on FVC\%

\begin{tabular}{|c|c|c|c|}
\hline Variables & $\mathrm{HR}$ & $95 \% \mathrm{Cl}$ & $P$ value \\
\hline ASA score & & & 0.998 \\
\hline I & 1.000 & & \\
\hline ॥ & 0.000 & 0.000 & 0.956 \\
\hline III & 0.000 & 0.000 & 0.974 \\
\hline \multicolumn{4}{|l|}{ Age } \\
\hline$<60$ & 1.000 & & \\
\hline $60-$ & 0.859 & $0.199-3.709$ & 0.839 \\
\hline \multicolumn{4}{|l|}{ Gender } \\
\hline Male & 1.000 & & \\
\hline Female & 0.425 & $0.045-4.036$ & 0.456 \\
\hline \multicolumn{4}{|l|}{ BMI } \\
\hline$<24.0 \mathrm{~kg} / \mathrm{m}^{2}$ & 1.000 & & \\
\hline$\geq 24.0 \mathrm{~kg} / \mathrm{m}^{2}$ & 0.518 & $0.093-2.893$ & 0.454 \\
\hline \multicolumn{4}{|l|}{ Smoking } \\
\hline No & 1.000 & & \\
\hline Yes & 4.045 & $0.577-28.338$ & 0.159 \\
\hline \multicolumn{4}{|l|}{ Diabetes mellitus } \\
\hline No & 1.000 & & \\
\hline Yes & 0.000 & 0.000 & 0.951 \\
\hline \multicolumn{4}{|l|}{ Coronary heart disease } \\
\hline No & 1.000 & & \\
\hline Yes & 0.000 & 0.000 & 0.995 \\
\hline \multicolumn{4}{|l|}{ Intraoperative bleeding } \\
\hline$<50 \mathrm{~mL}$ & 1.000 & & \\
\hline $50 \mathrm{~mL}-$ & 11.069 & $0.995-123.208$ & 0.051 \\
\hline \multicolumn{4}{|l|}{ Length of operation } \\
\hline$<2 \mathrm{~h}$ & 1.000 & & \\
\hline $2 \mathrm{~h}-$ & 1.272 & $0.226-7.149$ & 0.785 \\
\hline NSCLC staging & & & 0.137 \\
\hline IA & 1.000 & & \\
\hline IB & 0.000 & 0.000 & 0.983 \\
\hline IIA & 0.000 & 0.000 & 0.978 \\
\hline IIB & 0.000 & 0.000 & 0.998 \\
\hline IIIA & 14.556 & $1.997-106.085$ & 0.008 \\
\hline Postoperative pathology & & & 0.998 \\
\hline Adenocarcinoma & 1.000 & & \\
\hline Squamous cell carcinoma & 0.000 & 0.000 & 0.956 \\
\hline Adenosquamous carcinoma & 0.000 & 0.000 & 0.988 \\
\hline FVC \% & & & 0.559 \\
\hline$\leq 80.0 \%$ & 0.400 & $0.036-4.445$ & 0.455 \\
\hline$>80.0-92.0 \%$ & 1.268 & $0.247-6.520$ & 0.776 \\
\hline$>92.0-101.0 \%$ & 0.271 & $0.026-2.870$ & 0.278 \\
\hline$>101.0 \%$ & 1.000 & & \\
\hline
\end{tabular}

Results of binary logistics regression are presented as adjusted $\mathrm{HR}, 95 \% \mathrm{Cl}$ and $\mathrm{P}$ value. ASA, American Society of Anaesthesiologists; $\mathrm{BMI}$, body mass index; $\mathrm{Cl}$, confidence interval; FVC\%, forced vital capacity as percentage of predicted; HR, hazard ratio; NSCLC, nonsmall cell lung cancer. 
Table S15 Multivariate Cox regression results for overall survival based on FEV1\%

\begin{tabular}{|c|c|c|c|}
\hline Variables & $\mathrm{HR}$ & $95 \% \mathrm{Cl}$ & $P$ value \\
\hline ASA score & & & 0.999 \\
\hline I & 1.000 & & \\
\hline ॥ & 0.000 & 0.000 & 0.971 \\
\hline III & 0.000 & 0.000 & 0.977 \\
\hline \multicolumn{4}{|l|}{ Age } \\
\hline$<60$ & 1.000 & & \\
\hline $60-$ & 0.756 & $0.181-3.152$ & 0.701 \\
\hline \multicolumn{4}{|l|}{ Gender } \\
\hline Male & 1.000 & & \\
\hline Female & 0.496 & $0.049-4.991$ & 0.552 \\
\hline \multicolumn{4}{|l|}{ BMI } \\
\hline$<24.0 \mathrm{~kg} / \mathrm{m}^{2}$ & 1.000 & & \\
\hline$\geq 24.0 \mathrm{~kg} / \mathrm{m}^{2}$ & 0.623 & $0.120-3.227$ & 0.573 \\
\hline \multicolumn{4}{|l|}{ Smoking } \\
\hline No & 1.000 & & \\
\hline Yes & 3.867 & $0.509-29.355$ & 0.191 \\
\hline \multicolumn{4}{|l|}{ Diabetes mellitus } \\
\hline No & 1.000 & & \\
\hline Yes & 0.000 & 0.000 & 0.968 \\
\hline \multicolumn{4}{|l|}{ Coronary heart disease } \\
\hline No & 1.000 & & \\
\hline Yes & 0.000 & 0.000 & 0.998 \\
\hline \multicolumn{4}{|l|}{ Intraoperative bleeding } \\
\hline$<50 \mathrm{~mL}$ & 1.000 & & \\
\hline $50 \mathrm{~mL}-$ & 9.052 & $0.823-99.550$ & 0.072 \\
\hline \multicolumn{4}{|l|}{ Length of operation } \\
\hline$<2 \mathrm{~h}$ & 1.000 & & \\
\hline $2 \mathrm{~h}-$ & 1.618 & $0.298-8.768$ & 0.577 \\
\hline NSCLC staging & & & 0.090 \\
\hline IA & 1.000 & & \\
\hline IB & 0.000 & 0.000 & 0.981 \\
\hline IIA & 0.000 & 0.000 & 0.981 \\
\hline IIB & 0.000 & 0.000 & 0.997 \\
\hline IIIA & 15.354 & $2.321-101.557$ & 0.005 \\
\hline Postoperative pathology & & & 0.998 \\
\hline Adenocarcinoma & 1.000 & & \\
\hline Squamous cell carcinoma & 0.000 & 0.000 & 0.950 \\
\hline Adenosquamous carcinoma & 0.000 & 0.000 & 0.987 \\
\hline FEV1\% & & & 0.762 \\
\hline$\leq 78.4 \%$ & 0.860 & $0.125-5.917$ & 0.878 \\
\hline$>78.4-92.0 \%$ & 0.318 & $0.033-3.091$ & 0.324 \\
\hline$>92.0-103.0 \%$ & 0.555 & $0.091-3.404$ & 0.525 \\
\hline$>103.0 \%$ & 1.000 & & \\
\hline
\end{tabular}

Results of binary logistics regression are presented as adjusted $\mathrm{HR}, 95 \% \mathrm{Cl}$ and $\mathrm{P}$ value. ASA, American Society of Anaesthesiologists; $\mathrm{BMI}$, body mass index; $\mathrm{Cl}$, confidence interval; FEV1\%, forced expiratory volume in $1 \mathrm{~s}$ as percentage of predicted; HR, hazard ratio NSCLC, non-small cell lung cancer. 


\begin{tabular}{|c|c|c|c|c|c|}
\hline \multirow[b]{2}{*}{ Characteristic } & \multicolumn{5}{|c|}{ PEF } \\
\hline & $\begin{array}{c}\text { Quartile } 1(n=142) \\
\quad \leq 3.3 \mathrm{~L} / \mathrm{s}\end{array}$ & $\begin{array}{c}\text { Quartile } 2(n=136) \\
>3.3-4.4 \mathrm{~L} / \mathrm{s}\end{array}$ & $\begin{array}{c}\text { Quartile } 3(n=136) \\
\quad>4.4-5.8 \mathrm{~L} / \mathrm{s}\end{array}$ & $\begin{array}{c}\text { Quartile } 4(\mathrm{n}=134), \\
>5.8 \mathrm{~L} / \mathrm{s}\end{array}$ & $P$ value \\
\hline ASA score & & & & & 0.035 \\
\hline I & $114(80.3 \%)$ & $113(83.1 \%)$ & $123(90.4 \%)$ & $109(81.3 \%)$ & \\
\hline II & $16(11.3 \%)$ & $18(13.2 \%)$ & $11(8.1 \%)$ & $21(15.7 \%)$ & \\
\hline III & $12(8.5 \%)$ & $5(3.7 \%)$ & $2(1.5 \%)$ & $4(3.0 \%)$ & \\
\hline Age & $64.8 \pm 9.7$ & $64.6 \pm 10.5$ & $62.0 \pm 11.1$ & $60.4 \pm 10.6$ & 0.001 \\
\hline Gender (female/male) & $103 / 39$ & $86 / 50$ & $98 / 38$ & $44 / 90$ & 0.000 \\
\hline Weight, kg & $59.6 \pm 8.5$ & $62.2 \pm 10.8$ & $59.4 \pm 9.4$ & $64.4 \pm 9.5$ & 0.000 \\
\hline BMI, $\mathrm{kg} / \mathrm{m}^{2}$ & $23.3 \pm 2.6$ & $23.3 \pm 3.3$ & $22.7 \pm 2.9$ & $23.3 \pm 2.6$ & 0.125 \\
\hline Smoking & $33(23.2 \%)$ & $36(26.5 \%)$ & $26(19.1 \%)$ & $51(38.1 \%)$ & 0.003 \\
\hline Diabetes mellitus & $15(10.6 \%)$ & $12(8.8 \%)$ & $6(4.4 \%)$ & $15(11.2 \%)$ & 0.188 \\
\hline Coronary heart disease & $4(2.8 \%)$ & $2(1.5 \%)$ & $1(0.7 \%)$ & $2(1.5 \%)$ & 0.590 \\
\hline FEV1, L & $1.8 \pm 0.4$ & $2.2 \pm 0.5$ & $2.4 \pm 0.4$ & $2.9 \pm 0.5$ & 0.000 \\
\hline FVC, L & $2.5 \pm 0.6$ & $2.8 \pm 0.7$ & $2.9 \pm 0.5$ & $3.5 \pm 0.7$ & 0.000 \\
\hline PEF, L/s & $2.6 \pm 0.4$ & $3.9 \pm 0.3$ & $5.0 \pm 0.4$ & $7.3 \pm 1.2$ & 0.000 \\
\hline FEV1\% & $(78.5 \pm 13.6) \%$ & $(87.3 \pm 15.3) \%$ & $(97.7 \pm 17.1) \%$ & $(101.5 \pm 12.4) \%$ & 0.000 \\
\hline FVC\% & $(84.1 \pm 14.2) \%$ & $(87.1 \pm 15.4) \%$ & $(95.6 \pm 15.1) \%$ & $(98.7 \pm 13.4) \%$ & 0.000 \\
\hline PEF\% & $(42.2 \pm 9.0) \%$ & $(58.5 \pm 10.4) \%$ & $(76.4 \pm 13.1) \%$ & $(94.9 \pm 19.7) \%$ & 0.000 \\
\hline Intraoperative bleeding, $\mathrm{mL}$ & $49.4 \pm 41.4$ & $51.4 \pm 34.6$ & $45.3 \pm 27.5$ & $42.6 \pm 19.1$ & 0.682 \\
\hline Intraoperative blood transfusion, $\mathrm{mL}$ & 0 & 0 & 0 & 0 & - \\
\hline Length of operation, min & $124.8 \pm 37.0$ & $141.0 \pm 32.6$ & $131.0 \pm 35.9$ & $133.8 \pm 35.2$ & 0.002 \\
\hline NSCLC staging & & & & & 0.002 \\
\hline IA & $122(85.9 \%)$ & $99(72.8 \%)$ & $110(80.9 \%)$ & $116(86.6 \%)$ & \\
\hline IB & $4(2.8 \%)$ & $14(10.3 \%)$ & $12(8.2 \%)$ & $7(5.2 \%)$ & \\
\hline$\| \mathrm{A}$ & $4(2.8 \%)$ & $17(12.5 \%)$ & $6(4.4 \%)$ & $2(1.5 \%)$ & \\
\hline IIB & $1(0.7 \%)$ & $1(0.7 \%)$ & $0(0.0 \%)$ & $0(0.0 \%)$ & \\
\hline IIIA & $11(7.7 \%)$ & $5(3.7 \%)$ & $8(5.9 \%)$ & $9(6.7 \%)$ & \\
\hline Postoperative pathology & & & & & 0.891 \\
\hline Adenocarcinoma, $\mathrm{n}$ & $134(94.4 \%)$ & $123(90.4 \%)$ & $123(90.4 \%)$ & $124(92.5 \%)$ & \\
\hline Squamous cell carcinoma, $\mathrm{n}$ & $7(4.9 \%)$ & $11(8.1 \%)$ & $11(8.1 \%)$ & $8(6.0 \%)$ & \\
\hline Adenosquamous carcinoma, $\mathrm{n}$ & $1(0.7 \%)$ & $2(1.5 \%)$ & $2(1.5 \%)$ & $2(1.5 \%)$ & \\
\hline Postoperative length of stay, days & $5.4 \pm 1.7$ & $6.0 \pm 2.2$ & $5.9 \pm 1.8$ & $6.1 \pm 2.7$ & 0.032 \\
\hline Total hospital care costs (Renminbi) & $59532.8 \pm 10549.5$ & $59312.3 \pm 10203.7$ & $57275.2 \pm 8749.6$ & $57216.0 \pm 8927.6$ & 0.072 \\
\hline
\end{tabular}

Values are presented as mean \pm standard deviation, $\mathrm{n}$ or $\mathrm{n}(\%)$. ASA, American Society of Anaesthesiologists; BMI, body mass index; FEV1, forced expiratory volume in $1 \mathrm{~s}$; FEV1\%, FEV1 as percentage of predicted; FVC, forced vital capacity; FVC\%, FVC as percentage of predicted; NSCLC, non-small cell lung cancer; PEF, peak expiratory flow; PEF\%, PEF as percentage of predicted. 
Table S17 Univariate results of postoperative outcomes based on PEF

\begin{tabular}{|c|c|c|c|c|c|}
\hline \multirow[b]{2}{*}{ Postoperative outcome } & \multicolumn{5}{|c|}{ PEF } \\
\hline & $\begin{array}{l}\text { Quartile } 1(\mathrm{n}=142) \\
\quad \leq 3.3 \mathrm{~L} / \mathrm{s}\end{array}$ & $\begin{array}{c}\text { Quartile } 2(n=136) \\
>3.3-4.4 \mathrm{~L} / \mathrm{s}\end{array}$ & $\begin{array}{c}\text { Quartile } 3(n=136) \\
>4.4-5.8 \mathrm{~L} / \mathrm{s}\end{array}$ & $\begin{array}{c}\text { Quartile } 4(\mathrm{n}=134) \\
>5.8 \mathrm{~L} / \mathrm{s}\end{array}$ & $P$ value \\
\hline \multicolumn{6}{|l|}{ Postoperative pulmonary complications } \\
\hline Acute respiratory distress syndrome & $1(0.7 \%)$ & $2(1.5 \%)$ & $0(0.0 \%)$ & $1(0.7 \%)$ & 0.425 \\
\hline Reintubation & $0(0.0 \%)$ & $0(0.0 \%)$ & $0(0.0 \%)$ & $0(0.0 \%)$ & - \\
\hline Pulmonary embolism & $1(0.7 \%)$ & $2(1.5 \%)$ & $0(0.0 \%)$ & $0(0.0 \%)$ & 0.214 \\
\hline Need for bedside bronchoscopy & $0(0.0 \%)$ & $2(1.5 \%)$ & $0(0.0 \%)$ & $2(1.5 \%)$ & 0.128 \\
\hline Prolonged air leak & $1(0.7 \%)$ & $0(0.0 \%)$ & $3(2.2 \%)$ & $6(4.5 \%)$ & 0.017 \\
\hline Failure to expand & $4(2.8 \%)$ & $6(4.4 \%)$ & $0(0.0 \%)$ & $3(2.2 \%)$ & 0.035 \\
\hline Atelectasis & $0(0.0 \%)$ & $5(3.7 \%)$ & $7(5.1 \%)$ & $1(0.7 \%)$ & 0.004 \\
\hline Pneumonia & $62(43.7 \%)$ & $49(36.0 \%)$ & 54 (39.7\%) & $41(30.6 \%)$ & 0.143 \\
\hline Acute kidney injury & $2(1.4 \%)$ & $2(1.5 \%)$ & $2(1.5 \%)$ & $6(4.5 \%)$ & 0.292 \\
\hline In-hospital mortality, n & $0(0.0 \%)$ & $0(0.0 \%)$ & $0(0.0 \%)$ & $0(0.0 \%)$ & - \\
\hline Readmission within 30 days & $0(0.0 \%)$ & $2(1.5 \%)$ & $2(1.5 \%)$ & $2(1.5 \%)$ & 0.305 \\
\hline
\end{tabular}

Values are presented as $\mathrm{n}(\%)$. 
Table S18 Clinical characteristics of patients based on FVC

\begin{tabular}{|c|c|c|c|c|c|}
\hline \multirow[b]{2}{*}{ Characteristic } & \multicolumn{5}{|c|}{ FVC } \\
\hline & $\begin{array}{l}\text { Quartile } 1(n=122), \\
\leq 2.3 \mathrm{~L}\end{array}$ & $\begin{array}{c}\text { Quartile } 2(\mathrm{n}=170) \text {, } \\
>2.3-2.9 \mathrm{~L}\end{array}$ & $\begin{array}{c}\text { Quartile } 3(n=115) \text {, } \\
>2.9-3.4 \mathrm{~L}\end{array}$ & $\begin{array}{c}\text { Quartile } 4(\mathrm{n}=141), \\
>3.4 \mathrm{~L}\end{array}$ & $P$ value \\
\hline ASA score & & & & & 0.004 \\
\hline I & 89 (73.0\%) & 146 (85.9\%) & $102(88.7 \%)$ & $122(86.5 \%)$ & \\
\hline II & $24(19.7 \%)$ & $14(8.2 \%)$ & $11(9.6 \%)$ & $17(12.1 \%)$ & \\
\hline III & $9(7.4 \%)$ & $10(5.9 \%)$ & $2(1.7 \%)$ & $2(1.4 \%)$ & \\
\hline Age & $68.2 \pm 8.1$ & $62.7 \pm 10.9$ & $60.6 \pm 10.9$ & $60.8 \pm 10.4$ & 0.000 \\
\hline Gender (female/male) & $113 / 9$ & $137 / 33$ & $62 / 53$ & $19 / 122$ & 0.000 \\
\hline Weight, kg & $59.0 \pm 8.6$ & $58.3 \pm 8.9$ & $61.5 \pm 9.0$ & $66.9 \pm 10.0$ & 0.000 \\
\hline BMI, $\mathrm{kg} / \mathrm{m}^{2}$ & $23.7 \pm 3.0$ & $22.8 \pm 2.8$ & $22.9 \pm 2.6$ & $23.3 \pm 2.8$ & 0.032 \\
\hline Smoking & $7(5.7 \%)$ & $28(16.5 \%)$ & $35(30.4 \%)$ & $76(53.9 \%)$ & 0.000 \\
\hline Diabetes mellitus & $15(12.3 \%)$ & $12(7.1 \%)$ & $10(8.7 \%)$ & $11(7.8 \%)$ & 0.443 \\
\hline Coronary heart disease & $4(3.3 \%)$ & $3(1.8 \%)$ & $0(0.0 \%)$ & $2(1.4 \%)$ & 0.142 \\
\hline FEV1, L & $1.7 \pm 0.2$ & $2.1 \pm 0.3$ & $2.4 \pm 0.3$ & $3.0 \pm 0.4$ & 0.000 \\
\hline FVC, L & $2.0 \pm 0.2$ & $2.6 \pm 0.2$ & $3.1 \pm 0.1$ & $3.9 \pm 0.4$ & 0.000 \\
\hline PEF, L/s & $3.5 \pm 1.2$ & $4.3 \pm 1.5$ & $4.7 \pm 1.5$ & $6.1 \pm 2.1$ & 0.000 \\
\hline FEV1\% & $(78.4 \pm 11.6) \%$ & $(90.0 \pm 16.9) \%$ & $(95.6 \pm 18.3) \%$ & $(99.6 \pm 13.9) \%$ & 0.000 \\
\hline FVC\% & $(75.8 \pm 9.9) \%$ & $(89.7 \pm 12.9) \%$ & $(95.7 \pm 13.4) \%$ & $(103.0 \pm 12.8) \%$ & 0.000 \\
\hline PEF\% & $(59.7 \pm 21.5) \%$ & $(67.0 \pm 23.7) \%$ & $(68.7 \pm 23.6) \%$ & $(74.4 \pm 24.6) \%$ & 0.000 \\
\hline Intraoperative bleeding, mL & $52.1 \pm 45.0$ & $46.4 \pm 25.7$ & $47.0 \pm 31.7$ & $44.1 \pm 24.4$ & 0.231 \\
\hline Intraoperative blood transfusion, $\mathrm{mL}$ & 0 & 0 & 0 & 0 & - \\
\hline Length of operation, min & $131.1 \pm 38.4$ & $134.9 \pm 36.9$ & $131.7 \pm 33.0$ & $131.8 \pm 33.9$ & 0.781 \\
\hline NSCLC staging & & & & & 0.138 \\
\hline IA & $100(82.0 \%)$ & $139(81.8 \%)$ & $95(82.6 \%)$ & $113(80.1 \%)$ & \\
\hline IB & $11(9.0 \%)$ & $10(5.9 \%)$ & $9(7.8 \%)$ & $7(5.0 \%)$ & \\
\hline$\| \mathrm{A}$ & $6(4.9 \%)$ & $6(3.5 \%)$ & $9(7.8 \%)$ & $8(5.7 \%)$ & \\
\hline IIB & $1(0.8 \%)$ & $0(0.0 \%)$ & $0(0.0 \%)$ & $1(0.7 \%)$ & \\
\hline IIIA & $4(3.3 \%)$ & $15(8.8 \%)$ & $2(1.7 \%)$ & $12(8.5 \%)$ & \\
\hline Postoperative pathology & & & & & 0.101 \\
\hline Adenocarcinoma, $\mathrm{n}$ & $116(95.1 \%)$ & $156(91.8 \%)$ & $107(93.0 \%)$ & $125(88.7 \%)$ & \\
\hline Squamous cell carcinoma, $\mathrm{n}$ & $4(3.3 \%)$ & $10(5.9 \%)$ & $8(7.0 \%)$ & $15(10.6 \%)$ & \\
\hline Adenosquamous carcinoma, $\mathrm{n}$ & $2(1.6 \%)$ & $4(2.4 \%)$ & $0(0.0 \%)$ & $1(0.7 \%)$ & \\
\hline Postoperative length of stay, days & $5.8 \pm 2.5$ & $5.5 \pm 1.5$ & $5.9 \pm 2.0$ & $6.3 \pm 2.5$ & 0.027 \\
\hline Total hospital care costs (Renminbi) & $58689.2 \pm 11611.1$ & $57742.1 \pm 9218.8$ & $57714.8 \pm 8762.0$ & $59312.5 \pm 9124.4$ & 0.440 \\
\hline
\end{tabular}

Values are presented as mean \pm standard deviation, $n$ or $n$ (\%). ASA, American Society of Anaesthesiologists; BMI, body mass index; FEV1, forced expiratory volume in $1 \mathrm{~s}$; FEV1\%, FEV1 as percentage of predicted; FVC, forced vital capacity; FVC\%, FVC as percentage of predicted; NSCLC, non-small cell lung cancer; PEF, peak expiratory flow; PEF\%, PEF as percentage of predicted. 
Table S19 Univariate results of postoperative outcomes based on FVC

\begin{tabular}{|c|c|c|c|c|c|}
\hline \multirow[b]{2}{*}{ Postoperative outcome } & \multicolumn{5}{|c|}{ FVC } \\
\hline & $\begin{array}{l}\text { Quartile } 1(n=122) \\
\leq 2.3 \mathrm{~L}\end{array}$ & $\begin{array}{l}\text { Quartile } 2(\mathrm{n}=170) \\
\quad>2.3-2.9 \mathrm{~L}\end{array}$ & $\begin{array}{c}\text { Quartile } 3(\mathrm{n}=115) \\
>2.9-3.4 \mathrm{~L}\end{array}$ & $\begin{array}{c}\text { Quartile } 4(\mathrm{n}=141) \text {, } \\
>3.4 \mathrm{~L}\end{array}$ & $P$ value \\
\hline \multicolumn{6}{|l|}{ Postoperative pulmonary complications } \\
\hline Acute respiratory distress syndrome & $2(1.6 \%)$ & $0(0.0 \%)$ & $0(0.0 \%)$ & $2(1.4 \%)$ & 0.115 \\
\hline Reintubation & $0(0.0 \%)$ & $0(0.0 \%)$ & $0(0.0 \%)$ & $0(0.0 \%)$ & - \\
\hline Pulmonary embolism & $2(1.6 \%)$ & $0(0.0 \%)$ & $0(0.0 \%)$ & $1(0.7 \%)$ & 0.177 \\
\hline Need for bedside bronchoscopy & $0(0.0 \%)$ & $0(0.0 \%)$ & $1(0.9 \%)$ & $3(2.1 \%)$ & 0.078 \\
\hline Prolonged air leak & $0(0.0 \%)$ & $2(1.2 \%)$ & $1(0.9 \%)$ & $7(5.0 \%)$ & 0.012 \\
\hline Failure to expand & $2(1.6 \%)$ & $3(1.8 \%)$ & $4(3.5 \%)$ & $4(2.8 \%)$ & 0.736 \\
\hline Atelectasis & $5(4.1 \%)$ & $4(2.4 \%)$ & $2(1.7 \%)$ & $2(1.4 \%)$ & 0.540 \\
\hline Pneumonia & $61(50.0 \%)$ & $51(30.0 \%)$ & $53(46.1 \%)$ & $41(29.1 \%)$ & 0.000 \\
\hline Acute kidney injury & $2(1.6 \%)$ & $2(1.2 \%)$ & $3(2.6 \%)$ & $5(3.5 \%)$ & 0.517 \\
\hline In-hospital mortality, n & $0(0.0 \%)$ & $0(0.0 \%)$ & $0(0.0 \%)$ & $0(0.0 \%)$ & - \\
\hline Readmission within 30 days & $0(0.0 \%)$ & $1(0.6 \%)$ & $1(0.9 \%)$ & $4(2.8 \%)$ & 0.113 \\
\hline
\end{tabular}

Values are presented as $\mathrm{n}(\%)$. 
Table S20 Clinical characteristics of patients based on FEV1

\begin{tabular}{|c|c|c|c|c|c|}
\hline \multirow[t]{2}{*}{ Characteristic } & \multicolumn{5}{|c|}{ FEV1 } \\
\hline & $\begin{array}{l}\text { Quartile } 1(\mathrm{n}=122), \\
\leq 1.8 \mathrm{~L}\end{array}$ & $\begin{array}{c}\text { Quartile } 2(\mathrm{n}=167) \text {, } \\
>1.8-2.3 \mathrm{~L}\end{array}$ & $\begin{array}{c}\text { Quartile } 3(n=127) \text {, } \\
\quad>2.3-2.7 \mathrm{~L}\end{array}$ & $\begin{array}{c}\text { Quartile } 4(\mathrm{n}=132) \\
>2.7 \mathrm{~L}\end{array}$ & $P$ value \\
\hline ASA score & & & & & 0.006 \\
\hline I & $90(73.8 \%)$ & $138(82.6 \%)$ & $111(87.4 \%)$ & 120 (90.9\%) & \\
\hline II & $21(17.2 \%)$ & $23(13.8 \%)$ & $12(9.4 \%)$ & $10(7.6 \%)$ & \\
\hline III & $11(9.0 \%)$ & $6(3.6 \%)$ & $4(3.1 \%)$ & $2(1.5 \%)$ & \\
\hline Age & $68.4 \pm 8.4$ & $64.5 \pm 9.3$ & $59.6 \pm 11.7$ & $59.3 \pm 10.4$ & 0.000 \\
\hline Gender (female/male) & $98 / 24$ & $128 / 39$ & $85 / 42$ & $20 / 112$ & 0.000 \\
\hline Weight, kg & $59.2 \pm 9.9$ & $59.5 \pm 8.7$ & $60.2 \pm 8.6$ & $66.9 \pm 10.0$ & 0.000 \\
\hline $\mathrm{BMI}, \mathrm{kg} / \mathrm{m}^{2}$ & $23.4 \pm 3.2$ & $23.1 \pm 2.7$ & $22.8 \pm 2.7$ & $23.3 \pm 2.8$ & 0.301 \\
\hline Smoking & $19(15.6 \%)$ & $35(21.0 \%)$ & $27(21.3 \%)$ & $65(49.2 \%)$ & 0.000 \\
\hline Diabetes mellitus & $13(10.7 \%)$ & $17(10.2 \%)$ & $10(7.9 \%)$ & $8(6.1 \%)$ & 0.513 \\
\hline Coronary heart disease & $6(4.9 \%)$ & $1(0.6 \%)$ & $0(0.0 \%)$ & $2(1.5 \%)$ & 0.012 \\
\hline FEV1, L & $1.6 \pm 0.2$ & $2.0 \pm 0.1$ & $2.5 \pm 0.1$ & $3.1 \pm 0.3$ & 0.000 \\
\hline FVC, L & $2.2 \pm 0.4$ & $2.6 \pm 0.4$ & $3.1 \pm 0.4$ & $3.9 \pm 0.5$ & 0.000 \\
\hline PEF, L/s & $3.2 \pm 1.1$ & $4.0 \pm 1.3$ & $5.2 \pm 1.6$ & $6.4 \pm 1.9$ & 0.000 \\
\hline FEV1\% & $(72.0 \pm 10.7) \%$ & $(89.3 \pm 13.4) \%$ & $(99.9 \pm 14.4) \%$ & $(102.4 \pm 12.6) \%$ & 0.000 \\
\hline FVC\% & $(77.0 \pm 12.4) \%$ & $(89.0 \pm 12.1) \%$ & $(97.7 \pm 14.1) \%$ & $(101.2 \pm 13.1) \%$ & 0.000 \\
\hline PEF\% & $(51.9 \pm 16.6) \%$ & $(64.0 \pm 22.3) \%$ & $(76.7 \pm 23.7) \%$ & $(78.0 \pm 23.1) \%$ & 0.000 \\
\hline Intraoperative bleeding, $\mathrm{mL}$ & $50.3 \pm 45.5$ & $50.9 \pm 29.3$ & $43.5 \pm 26.4$ & $43.2 \pm 23.2$ & 0.067 \\
\hline Intraoperative blood transfusion, $\mathrm{mL}$ & 0 & 0 & 0 & 0 & - \\
\hline Length of operation, min & $130.6 \pm 38.1$ & $133.4 \pm 35.9$ & $134.5 \pm 32.0$ & $131.5 \pm 36.4$ & 0.818 \\
\hline NSCLC staging & & & & & 0.000 \\
\hline $\mathrm{IA}$ & $89(73.0 \%)$ & $144(86.2 \%)$ & $113(89.0 \%)$ & $101(76.5 \%)$ & \\
\hline IB & $18(14.8 \%)$ & $9(5.4 \%)$ & $2(1.6 \%)$ & $8(6.1 \%)$ & \\
\hline IIA & $6(4.9 \%)$ & $5(3.0 \%)$ & $10(7.9 \%)$ & $8(6.1 \%)$ & \\
\hline IIB & $0(0.0 \%)$ & $1(0.6 \%)$ & $0(0.0 \%)$ & $1(0.8 \%)$ & \\
\hline IIIA & $9(7.4 \%)$ & $8(4.8 \%)$ & $2(1.6 \%)$ & $14(10.6 \%)$ & \\
\hline Postoperative pathology & & & & & 0.369 \\
\hline Adenocarcinoma, $\mathrm{n}$ & $111(91.0 \%)$ & $154(92.2 \%)$ & $120(94.5 \%)$ & $119(90.2 \%)$ & \\
\hline Squamous cell carcinoma, $\mathrm{n}$ & $8(6.6 \%)$ & $10(6.0 \%)$ & $7(5.5 \%)$ & $12(9.1 \%)$ & \\
\hline Adenosquamous carcinoma, $\mathrm{n}$ & $3(2.5 \%)$ & $3(1.8 \%)$ & $0(0.0 \%)$ & $1(0.8 \%)$ & \\
\hline Postoperative length of stay, days & $5.5 \pm 2.3$ & $5.8 \pm 1.6$ & $5.8 \pm 2.0$ & $6.2 \pm 2.6$ & 0.035 \\
\hline Total hospital care costs (Renminbi) & $58686.9 \pm 11793.0$ & $58427.9 \pm 9387.9$ & $56796.9 \pm 7115.5$ & $59439.7 \pm 9970.1$ & 0.267 \\
\hline
\end{tabular}

Values are presented as mean \pm standard deviation, $n$ or $n$ (\%). ASA, American Society of Anaesthesiologists; BMI, body mass index; FEV1, forced expiratory volume in $1 \mathrm{~s}$; FEV1\%, FEV1 as percentage of predicted; FVC, forced vital capacity; FVC\%, FVC as percentage of predicted; NSCLC, non-small cell lung cancer; PEF, peak expiratory flow; PEF\%, PEF as percentage of predicted. 
Table S21 Univariate results of postoperative outcomes based on FEV1

\begin{tabular}{|c|c|c|c|c|c|}
\hline \multirow[b]{2}{*}{ Postoperative outcome } & \multicolumn{5}{|c|}{ FEV1 } \\
\hline & $\begin{array}{c}\text { Quartile } 1(\mathrm{n}=122), \\
\leq 1.8 \mathrm{~L}\end{array}$ & $\begin{array}{c}\text { Quartile } 2(\mathrm{n}=167) \\
>1.8-2.3 \mathrm{~L}\end{array}$ & $\begin{array}{c}\text { Quartile } 3(n=127) \\
>2.3-2.7 \mathrm{~L}\end{array}$ & $\begin{array}{c}\text { Quartile } 4(\mathrm{n}=132) \text {, } \\
>2.7 \mathrm{~L}\end{array}$ & $P$ value \\
\hline \multicolumn{6}{|l|}{ Postoperative pulmonary complications } \\
\hline Acute respiratory distress syndrome & $0(0.0 \%)$ & $2(1.2 \%)$ & $0(0.0 \%)$ & $2(1.5 \%)$ & 0.177 \\
\hline Reintubation & $0(0.0 \%)$ & $0(0.0 \%)$ & $0(0.0 \%)$ & $0(0.0 \%)$ & - \\
\hline Pulmonary embolism & $0(0.0 \%)$ & $2(1.2 \%)$ & $0(0.0 \%)$ & $1(0.8 \%)$ & 0.284 \\
\hline Need for bedside bronchoscopy & $0(0.0 \%)$ & $2(1.2 \%)$ & $1(0.8 \%)$ & $1(0.8 \%)$ & 0.529 \\
\hline Prolonged air leak & $0(0.0 \%)$ & $0(0.0 \%)$ & $6(4.7 \%)$ & $4(3.0 \%)$ & 0.001 \\
\hline Failure to expand & $2(1.6 \%)$ & $6(3.6 \%)$ & $0(0.0 \%)$ & $5(3.8 \%)$ & 0.040 \\
\hline Atelectasis & $2(1.6 \%)$ & $5(3.0 \%)$ & $5(3.9 \%)$ & $1(0.8 \%)$ & 0.294 \\
\hline Pneumonia & $52(42.6 \%)$ & $61(36.5 \%)$ & $46(36.2 \%)$ & $47(35.6 \%)$ & 0.632 \\
\hline Acute kidney injury & $2(1.6 \%)$ & $2(1.2 \%)$ & $4(3.1 \%)$ & $4(3.0 \%)$ & 0.577 \\
\hline In-hospital mortality, n & $0(0.0 \%)$ & $0(0.0 \%)$ & $0(0.0 \%)$ & $0(0.0 \%)$ & - \\
\hline Readmission within 30 days & $0(0.0 \%)$ & $0(0.0 \%)$ & $1(0.8 \%)$ & $5(3.8 \%)$ & 0.008 \\
\hline
\end{tabular}

Values are presented as $n(\%)$.

Table S22 Effect of PEF on postoperative outcomes

\begin{tabular}{|c|c|c|c|c|c|c|}
\hline \multirow{3}{*}{ Postoperative outcome } & & \multicolumn{4}{|c|}{ PEF } & \multirow{3}{*}{$\begin{array}{c}\text { Total } \\
\text { P value }\end{array}$} \\
\hline & & \multicolumn{4}{|c|}{ Quartile 1 ( $n=142)$, Quartile $2(n=136)$, Quartile $3(n=136)$, Quartile $4(n=134)$, } & \\
\hline & & $\leq 3.3 \mathrm{~L} / \mathrm{s}$ & $>3.3-4.4 \mathrm{~L} / \mathrm{s}$ & $>4.4-5.8 \mathrm{~L} / \mathrm{s}$ & $>5.8 \mathrm{~L} / \mathrm{s}$ & \\
\hline \multicolumn{7}{|l|}{ Postoperative pulmonary complications } \\
\hline Acute respiratory distress syndrome & $\mathrm{N}(\%)$ & $1(0.7 \%)$ & $2(1.5 \%)$ & $0(0.0 \%)$ & $1(0.7 \%)$ & 0.966 \\
\hline Reintubation & $N(\%)$ & $0(0.0 \%)$ & $0(0.0 \%)$ & $0(0.0 \%)$ & $0(0.0 \%)$ & - \\
\hline Pulmonary embolism & $\mathrm{N}(\%)$ & $1(0.7 \%)$ & $2(1.5 \%)$ & $0(0.0 \%)$ & $0(0.0 \%)$ & 1.000 \\
\hline Need for bedside bronchoscopy & $\mathrm{N}(\%)$ & $0(0.0 \%)$ & $2(1.5 \%)$ & $0(0.0 \%)$ & $2(1.5 \%)$ & 1.000 \\
\hline Prolonged air leak & $N(\%)$ & $1(0.7 \%)$ & $0(0.0 \%)$ & $3(2.2 \%)$ & $6(4.5 \%)$ & 0.756 \\
\hline Failure to expand & $N(\%)$ & $4(2.8 \%)$ & $6(4.4 \%)$ & $0(0.0 \%)$ & $3(2.2 \%)$ & 0.822 \\
\hline Atelectasis & $\mathrm{N}(\%)$ & $0(0.0 \%)$ & $5(3.7 \%)$ & $7(5.1 \%)$ & $1(0.7 \%)$ & 0.581 \\
\hline Pneumonia & $\begin{array}{l}\text { N (\%), OR } \\
(95 \% \mathrm{Cl}) \\
\text { P value }\end{array}$ & $\begin{array}{c}62(43.7 \%), 1.661 \\
(0.939-2.939) \\
P=0.081\end{array}$ & $\begin{array}{c}49(36.0 \%), 0.940 \\
(0.527-1.675), \\
P=0.833\end{array}$ & $\begin{array}{c}54(39.7 \%), 1.391 \\
(0.793-2.442) \\
P=0.250\end{array}$ & $41(30.6 \%), 1$ & 0.130 \\
\hline Acute kidney injury & N (\%) & $2(1.4 \%)$ & $2(1.5 \%)$ & $2(1.5 \%)$ & $6(4.5 \%)$ & 0.521 \\
\hline In-hospital mortality & $\mathrm{N}(\%)$ & $0(0.0 \%)$ & $0(0.0 \%)$ & $0(0.0 \%)$ & $0(0.0 \%)$ & - \\
\hline Readmission within 30 days & $\mathrm{N}(\%)$ & $0(0.0 \%)$ & $2(1.5 \%)$ & $2(1.5 \%)$ & $2(1.5 \%)$ & 0.905 \\
\hline
\end{tabular}

Results of binary logistics regression are presented as adjusted $\mathrm{OR}, 95 \% \mathrm{Cl}$ and $\mathrm{P}$ value. The best-performing quartile 4 served as the reference group. $\mathrm{Cl}$, confidence interval; OR, odds ratio; PEF, peak expiratory flow. 
Table S23 Full-model multivariate results of 4 groups of different PEF

\begin{tabular}{|c|c|c|c|}
\hline \multirow{2}{*}{ Variables } & \multicolumn{3}{|c|}{ Postoperative pneumonia } \\
\hline & OR & $95 \% \mathrm{Cl}$ & $P$ value \\
\hline ASA score & & & 0.014 \\
\hline I & 1.000 & & \\
\hline II & 3.373 & $1.437-7.917$ & 0.005 \\
\hline III & 2.838 & $0.743-10.838$ & 0.127 \\
\hline \multicolumn{4}{|l|}{ Age } \\
\hline$<60$ & 1.000 & & \\
\hline $60-$ & 1.339 & $0.889-2.016$ & 0.162 \\
\hline \multicolumn{4}{|l|}{ Gender } \\
\hline Male & 1.000 & & \\
\hline Female & 1.175 & $0.648-2.130$ & 0.596 \\
\hline \multicolumn{4}{|l|}{ BMI } \\
\hline$<24.0 \mathrm{~kg} / \mathrm{m}^{2}$ & 1.000 & & \\
\hline$\geq 24.0 \mathrm{~kg} / \mathrm{m}^{2}$ & 1.620 & $1.089-2.410$ & 0.017 \\
\hline \multicolumn{4}{|l|}{ Smoking } \\
\hline No & 1.000 & & \\
\hline Yes & 0.953 & $0.495-1.837$ & 0.886 \\
\hline \multicolumn{4}{|l|}{ Diabetes mellitus } \\
\hline No & 1.000 & & \\
\hline Yes & 0.105 & $0.037-0.304$ & 0.000 \\
\hline \multicolumn{4}{|l|}{ Coronary heart disease } \\
\hline No & 1.000 & & \\
\hline Yes & 0.467 & $0.068-3.196$ & 0.438 \\
\hline \multicolumn{4}{|l|}{ Intraoperative bleeding } \\
\hline$<50 \mathrm{~mL}$ & 1.000 & & \\
\hline $50 \mathrm{~mL}-$ & 0.982 & $0.659-1.463$ & 0.928 \\
\hline \multicolumn{4}{|l|}{ Length of operation } \\
\hline$<2 \mathrm{~h}$ & 1.000 & & \\
\hline $2 \mathrm{~h}-$ & 1.652 & $1.095-2.490$ & 0.017 \\
\hline NSCLC staging & & & 0.450 \\
\hline IA & 1.000 & & \\
\hline IB & 0.917 & $0.428-1.966$ & 0.824 \\
\hline$\| \mathrm{A}$ & 2.219 & $0.946-5.209$ & 0.067 \\
\hline IIB & 1.932 & $0.111-33.650$ & 0.651 \\
\hline IIIA & 1.080 & $0.494-2.363$ & 0.847 \\
\hline Postoperative pathology & & & 0.177 \\
\hline Adenocarcinoma & 1.000 & & \\
\hline Squamous cell carcinoma & 2.134 & $0.961-4.740$ & 0.063 \\
\hline Adenosquamous carcinoma & 0.000 & 0.000 & 0.999 \\
\hline PEF, L/s & & & 0.130 \\
\hline$\leq 3.3 \mathrm{~L} / \mathrm{s}$ & 1.661 & $0.939-2.939$ & 0.081 \\
\hline$>3.3-4.4 \mathrm{~L} / \mathrm{s}$ & 0.940 & $0.527-1.675$ & 0.833 \\
\hline$>4.4-5.8 \mathrm{~L} / \mathrm{s}$ & 1.391 & $0.793-2.442$ & 0.250 \\
\hline$>5.8 \mathrm{~L} / \mathrm{s}$ & 1.000 & & \\
\hline
\end{tabular}

Results of binary logistics regression are presented as adjusted OR, 95\% Cl and P value. ASA, American Society of Anaesthesiologists $\mathrm{BMI}$, body mass index; $\mathrm{Cl}$, confidence interval; NSCLC, non-small cell lung cancer; OR, odds ratio; PEF, peak expiratory flow. 
Table S24 Effect of FVC on postoperative outcomes

\begin{tabular}{|c|c|c|c|c|c|c|}
\hline \multirow[t]{3}{*}{ Postoperative outcome } & & \multicolumn{4}{|c|}{ FVC } & \multirow{3}{*}{$\begin{array}{c}\text { Total } \\
\text { P value }\end{array}$} \\
\hline & & \multicolumn{4}{|c|}{ Quartile 1 ( $n=122)$, Quartile $2(n=170)$, Quartile $3(n=115)$, Quartile $4(n=141)$, } & \\
\hline & & $\leq 2.3 \mathrm{~L}$ & $>2.3-2.9 \mathrm{~L}$ & $>2.9-3.4 \mathrm{~L}$ & $>3.4 \mathrm{~L}$ & \\
\hline \multicolumn{7}{|l|}{ Postoperative pulmonary complications } \\
\hline Acute respiratory distress syndrome & $\mathrm{N}(\%)$ & $2(1.6 \%)$ & $0(0.0 \%)$ & $0(0.0 \%)$ & $2(1.4 \%)$ & 0.817 \\
\hline Reintubation & $\mathrm{N}(\%)$ & $0(0.0 \%)$ & $0(0.0 \%)$ & $0(0.0 \%)$ & $0(0.0 \%)$ & - \\
\hline Pulmonary embolism & $\mathrm{N}(\%)$ & $2(1.6 \%)$ & $0(0.0 \%)$ & $0(0.0 \%)$ & $1(0.7 \%)$ & 1.000 \\
\hline Need for bedside bronchoscopy & $\mathrm{N}(\%)$ & $0(0.0 \%)$ & $0(0.0 \%)$ & $1(0.9 \%)$ & $3(2.1 \%)$ & 0.996 \\
\hline Prolonged air leak & $\mathrm{N}(\%)$ & $0(0.0 \%)$ & $2(1.2 \%)$ & $1(0.9 \%)$ & $7(5.0 \%)$ & 0.714 \\
\hline Failure to expand & $\mathrm{N}(\%)$ & $2(1.6 \%)$ & $3(1.8 \%)$ & $4(3.5 \%)$ & $4(2.8 \%)$ & 0.572 \\
\hline Atelectasis & $\mathrm{N}(\%)$ & $5(4.1 \%)$ & $4(2.4 \%)$ & $2(1.7 \%)$ & $2(1.4 \%)$ & 0.363 \\
\hline Pneumonia & $\begin{array}{c}\text { N (\%), OR } \\
(95 \% \mathrm{Cl}) \\
\text { P value }\end{array}$ & $\begin{array}{c}61(50.0 \%) \\
2.904 \\
(1.397-6.035) \\
P=0.004\end{array}$ & $\begin{array}{c}51(30.0 \%) \\
1.173 \\
(0.619-2.225) \\
P=0.625\end{array}$ & $\begin{array}{c}53(46.1 \%) \\
2.582 \\
(1.432-4.659), \\
P=0.002\end{array}$ & $41(29.1 \%), 1$ & 0.000 \\
\hline Acute kidney injury & $\mathrm{N}(\%)$ & $2(1.6 \%)$ & $2(1.2 \%)$ & $3(2.6 \%)$ & $5(3.5 \%)$ & 0.838 \\
\hline In-hospital mortality & $\mathrm{N}(\%)$ & $0(0.0 \%)$ & $0(0.0 \%)$ & $0(0.0 \%)$ & $0(0.0 \%)$ & - \\
\hline Readmission within 30 days & $\mathrm{N}(\%)$ & $0(0.0 \%)$ & $1(0.6 \%)$ & $1(0.9 \%)$ & $4(2.8 \%)$ & 0.913 \\
\hline
\end{tabular}

Results of binary logistics regression are presented as adjusted $\mathrm{OR}, 95 \% \mathrm{Cl}$ and $\mathrm{P}$ value. The best-performing quartile 4 served as the reference group. $\mathrm{Cl}$, confidence interval; FVC, forced vital capacity; OR, odds ratio. 
Table S25 Full-model multivariate results of 4 groups of different FVC

\begin{tabular}{|c|c|c|c|}
\hline \multirow{2}{*}{ Variables } & \multicolumn{3}{|c|}{ Postoperative pneumonia } \\
\hline & OR & $95 \% \mathrm{Cl}$ & $P$ value \\
\hline ASA score & & & 0.022 \\
\hline I & 1.000 & & \\
\hline II & 3.017 & $1.269-7.174$ & 0.012 \\
\hline III & 3.282 & $0.865-12.459$ & 0.081 \\
\hline \multicolumn{4}{|l|}{ Age } \\
\hline$<60$ & 1.000 & & \\
\hline $60-$ & 1.262 & $0.822-1.937$ & 0.288 \\
\hline \multicolumn{4}{|l|}{ Gender } \\
\hline Male & 1.000 & & \\
\hline Female & 0.947 & $0.475-1.888$ & 0.878 \\
\hline \multicolumn{4}{|l|}{ BMI } \\
\hline$<24.0 \mathrm{~kg} / \mathrm{m}^{2}$ & 1.000 & & \\
\hline$\geq 24.0 \mathrm{~kg} / \mathrm{m}^{2}$ & 1.497 & $1.000-2.243$ & 0.050 \\
\hline \multicolumn{4}{|l|}{ Smoking } \\
\hline No & 1.000 & & \\
\hline Yes & 0.981 & $0.507-1.897$ & 0.954 \\
\hline \multicolumn{4}{|l|}{ Diabetes mellitus } \\
\hline No & 1.000 & & \\
\hline Yes & 0.094 & $0.032-0.275$ & 0.000 \\
\hline \multicolumn{4}{|l|}{ Coronary heart disease } \\
\hline No & 1.000 & & \\
\hline Yes & 0.452 & $0.069-2.975$ & 0.409 \\
\hline \multicolumn{4}{|l|}{ Intraoperative bleeding } \\
\hline$<50 \mathrm{~mL}$ & 1.000 & & \\
\hline $50 \mathrm{~mL}-$ & 0.957 & $0.638-1.436$ & 0.833 \\
\hline \multicolumn{4}{|l|}{ Length of operation } \\
\hline$<2 \mathrm{~h}$ & 1.000 & & \\
\hline $2 \mathrm{~h}-$ & 1.618 & $1.072-2.442$ & 0.022 \\
\hline NSCLC staging & & & 0.431 \\
\hline IA & 1.000 & & \\
\hline IB & 0.783 & $0.362-1.695$ & 0.535 \\
\hline$\| \mathrm{A}$ & 1.991 & $0.856-4.635$ & 0.110 \\
\hline IIB & 1.735 & $0.098-30.758$ & 0.707 \\
\hline IIIA & 1.413 & $0.636-3.141$ & 0.396 \\
\hline Postoperative pathology & & & 0.156 \\
\hline Adenocarcinoma & 1.000 & & \\
\hline Squamous cell carcinoma & 2.220 & $0.986-4.999$ & 0.054 \\
\hline Adenosquamous carcinoma & 0.000 & 0.000 & 0.999 \\
\hline FVC, L & & & 0.000 \\
\hline$\leq 2.3 \mathrm{~L}$ & 2.904 & $1.397-6.035$ & 0.004 \\
\hline$>2.3-2.9 \mathrm{~L}$ & 1.173 & $0.619-2.225$ & 0.625 \\
\hline$>2.9-3.4 \mathrm{~L}$ & 2.582 & $1.432-4.659$ & 0.002 \\
\hline$>3.4 \mathrm{~L}$ & 1.000 & & \\
\hline
\end{tabular}

Results of binary logistics regression are presented as adjusted OR, 95\% Cl and P value. ASA, American Society of Anaesthesiologists; $\mathrm{BMI}$, body mass index; $\mathrm{Cl}$, confidence interval; FVC, forced vital capacity; NSCLC, non-small cell lung cancer; OR, odds ratio. 
Table S26 Effect of FEV1 on postoperative outcomes

\begin{tabular}{|c|c|c|c|c|c|c|}
\hline \multirow{3}{*}{ Postoperative outcome } & \multicolumn{5}{|c|}{ FEV1 } & \multirow{3}{*}{$\begin{array}{c}\text { Total } \\
\text { P value }\end{array}$} \\
\hline & & \multicolumn{4}{|c|}{ Quartile $1(n=122)$, Quartile $2(n=167)$, Quartile $3(n=127)$, Quartile $4(n=132)$, } & \\
\hline & & $\leq 1.8 \mathrm{~L}$ & $>1.8-2.3 \mathrm{~L}$ & $>2.3-2.7 \mathrm{~L}$ & $>2.7 \mathrm{~L}$ & \\
\hline \multicolumn{7}{|l|}{ Postoperative pulmonary complications } \\
\hline Acute respiratory distress syndrome & $\mathrm{N}(\%)$ & $0(0.0 \%)$ & $2(1.2 \%)$ & $0(0.0 \%)$ & $2(1.5 \%)$ & 0.773 \\
\hline Reintubation & $\mathrm{N}(\%)$ & $0(0.0 \%)$ & $0(0.0 \%)$ & $0(0.0 \%)$ & $0(0.0 \%)$ & - \\
\hline Pulmonary embolism & $\mathrm{N}(\%)$ & $0(0.0 \%)$ & $2(1.2 \%)$ & $0(0.0 \%)$ & $1(0.8 \%)$ & 0.962 \\
\hline Need for bedside bronchoscopy & $\mathrm{N}(\%)$ & $0(0.0 \%)$ & $2(1.2 \%)$ & $1(0.8 \%)$ & $1(0.8 \%)$ & 0.452 \\
\hline Prolonged air leak & $\mathrm{N}(\%)$ & $0(0.0 \%)$ & $0(0.0 \%)$ & $6(4.7 \%)$ & $4(3.0 \%)$ & 0.308 \\
\hline Failure to expand & $\mathrm{N}(\%)$ & $2(1.6 \%)$ & $6(3.6 \%)$ & $0(0.0 \%)$ & $5(3.8 \%)$ & 0.545 \\
\hline Atelectasis & $\mathrm{N}(\%)$ & $2(1.6 \%)$ & $5(3.0 \%)$ & $5(3.9 \%)$ & $1(0.8 \%)$ & 0.331 \\
\hline Pneumonia & $\begin{array}{c}\mathrm{N}(\%), \mathrm{OR} \\
(95 \% \mathrm{Cl}) \\
\mathrm{P} \text { value }\end{array}$ & $\begin{array}{c}52(42.6 \%) \\
1.027 \\
(0.523-2.016) \\
P=0.938\end{array}$ & $\begin{array}{c}61(36.5 \%), \\
0.878 \\
(0.475-1.622), \\
P=0.677\end{array}$ & $\begin{array}{c}46(36.2 \%) \\
0.954 \\
(0.524-1.736) \\
P=0.876\end{array}$ & 47 (35.6\%), 1 & 0.939 \\
\hline Acute kidney injury & $\mathrm{N}(\%)$ & $2(1.6 \%)$ & $2(1.2 \%)$ & $4(3.1 \%)$ & $4(3.0 \%)$ & 0.328 \\
\hline In-hospital mortality & $N(\%)$ & $0(0.0 \%)$ & $0(0.0 \%)$ & $0(0.0 \%)$ & $0(0.0 \%)$ & - \\
\hline Readmission within 30 days & $\mathrm{N}(\%)$ & $0(0.0 \%)$ & $0(0.0 \%)$ & $1(0.8 \%)$ & $5(3.8 \%)$ & 0.666 \\
\hline
\end{tabular}

Results of binary logistics regression are presented as adjusted $\mathrm{OR}, 95 \% \mathrm{Cl}$ and $\mathrm{P}$ value. The best-performing quartile 4 served as the reference group. $\mathrm{Cl}$, confidence interval; FEV1, forced expiratory volume in $1 \mathrm{~s}$; OR, odds ratio. 
Table S27 Full-model multivariate results of 4 groups of different FEV1

\begin{tabular}{|c|c|c|c|}
\hline \multirow{2}{*}{ Variables } & \multicolumn{3}{|c|}{ Postoperative pneumonia } \\
\hline & OR & $95 \% \mathrm{Cl}$ & $P$ value \\
\hline ASA score & & & 0.019 \\
\hline I & 1.000 & & \\
\hline II & 3.173 & $1.345-7.487$ & 0.008 \\
\hline III & 2.917 & $0.769-11.060$ & 0.116 \\
\hline \multicolumn{4}{|l|}{ Age } \\
\hline$<60$ & 1.000 & & \\
\hline $60-$ & 1.405 & $0.920-2.147$ & 0.116 \\
\hline \multicolumn{4}{|l|}{ Gender } \\
\hline Male & 1.000 & & \\
\hline Female & 1.404 & $0.724-2.722$ & 0.315 \\
\hline \multicolumn{4}{|l|}{ BMI } \\
\hline$<24.0 \mathrm{~kg} / \mathrm{m}^{2}$ & 1.000 & & \\
\hline$\geq 24.0 \mathrm{~kg} / \mathrm{m}^{2}$ & 1.565 & $1.050-2.331$ & 0.028 \\
\hline \multicolumn{4}{|l|}{ Smoking } \\
\hline No & 1.000 & & \\
\hline Yes & 1.024 & $0.532-1.972$ & 0.944 \\
\hline \multicolumn{4}{|l|}{ Diabetes mellitus } \\
\hline No & 1.000 & & \\
\hline Yes & 0.112 & $0.039-0.324$ & 0.000 \\
\hline \multicolumn{4}{|l|}{ Coronary heart disease } \\
\hline No & 1.000 & & \\
\hline Yes & 0.454 & $0.067-3.077$ & 0.418 \\
\hline \multicolumn{4}{|l|}{ Intraoperative bleeding } \\
\hline$<50 \mathrm{~mL}$ & 1.000 & & \\
\hline $50 \mathrm{~mL}-$ & 0.993 & $0.666-1.481$ & 0.973 \\
\hline \multicolumn{4}{|l|}{ Length of operation } \\
\hline$<2 \mathrm{~h}$ & 1.000 & & \\
\hline $2 \mathrm{~h}-$ & 1.525 & $1.020-2.280$ & 0.040 \\
\hline NSCLC staging & & & 0.604 \\
\hline IA & 1.000 & & \\
\hline IB & 0.849 & $0.393-1.837$ & 0.678 \\
\hline$\| \mathrm{A}$ & 1.915 & $0.825-4.444$ & 0.130 \\
\hline IIB & 1.926 & $0.113-32.741$ & 0.650 \\
\hline IIIA & 1.071 & $0.490-2.340$ & 0.863 \\
\hline Postoperative pathology & & & 0.147 \\
\hline Adenocarcinoma & 1.000 & & \\
\hline Squamous cell carcinoma & 2.222 & $0.999-4.940$ & 0.050 \\
\hline Adenosquamous carcinoma & 0.000 & 0.000 & 0.999 \\
\hline FEV1, L & & & 0.939 \\
\hline$\leq 1.8 \mathrm{~L}$ & 1.027 & $0.523-2.016$ & 0.938 \\
\hline$>1.8-2.3 \mathrm{~L}$ & 0.878 & $0.475-1.622$ & 0.677 \\
\hline$>2.3-2.7 \mathrm{~L}$ & 0.954 & $0.524-1.736$ & 0.876 \\
\hline$>2.7 \mathrm{~L}$ & 1.000 & & \\
\hline
\end{tabular}

Results of binary logistics regression are presented as adjusted OR, 95\% Cl and P value. ASA, American Society of Anaesthesiologists $\mathrm{BMI}$, body mass index; $\mathrm{Cl}$, confidence interval; FEV1, forced expiratory volume in $1 \mathrm{~s}$; NSCLC, non-small cell lung cancer; OR, odds ratio. 
Table S28 Mortality following video-assisted thoracic surgery lobectomy for lung cancer based on PEF

\begin{tabular}{|c|c|c|c|c|c|}
\hline \multirow[b]{2}{*}{ Variables } & \multicolumn{5}{|c|}{ PEF } \\
\hline & $\begin{array}{c}\text { Quartile } 1(n=142), \\
\leq 3.3 \mathrm{~L} / \mathrm{s}\end{array}$ & $\begin{array}{c}\text { Quartile } 2(\mathrm{n}=136) \\
>3.3-4.4 \mathrm{~L} / \mathrm{s}\end{array}$ & $\begin{array}{c}\text { Quartile } 3(\mathrm{n}=136) \\
>4.4-5.8 \mathrm{~L} / \mathrm{s}\end{array}$ & $\begin{array}{c}\text { Quartile } 4(\mathrm{n}=134) \\
>5.8 \mathrm{~L} / \mathrm{s}\end{array}$ & $P$ value \\
\hline Number of deaths & $2(1.4 \%)$ & $0(0.0 \%)$ & $1(0.7 \%)$ & $6(4.5 \%)$ & 0.019 \\
\hline Cause of death & & & & & 0.376 \\
\hline Postoperative complication & $0(0.0 \%)$ & $0(0.0 \%)$ & $0(0.0 \%)$ & $0(0.0 \%)$ & \\
\hline Cancer related & $1(50.0 \%)$ & $0(0.0 \%)$ & $0(0.0 \%)$ & $4(66.7 \%)$ & \\
\hline Non-cancer related & $0(0.0 \%)$ & $0(0.0 \%)$ & $0(0.0 \%)$ & $0(0.0 \%)$ & \\
\hline Uncertain & $1(50.0 \%)$ & $0(0.0 \%)$ & $1(100.0 \%)$ & $2(33.3 \%)$ & \\
\hline
\end{tabular}

Values are presented as $\mathrm{n}(\%)$. PEF, peak expiratory flow.

Table S29 Mortality following video-assisted thoracic surgery lobectomy for lung cancer based on FVC

\begin{tabular}{|c|c|c|c|c|c|}
\hline \multirow[b]{2}{*}{ Variables } & \multicolumn{5}{|c|}{ FVC } \\
\hline & $\begin{array}{l}\text { Quartile } 1(n=122), \\
\leq 2.3 \mathrm{~L}\end{array}$ & $\begin{array}{c}\text { Quartile } 2(\mathrm{n}=170) \\
>2.3 \mathrm{~L}-2.9 \mathrm{~L}\end{array}$ & $\begin{array}{c}\text { Quartile } 3(\mathrm{n}=115) \\
>2.9 \mathrm{~L}-3.4 \mathrm{~L}\end{array}$ & $\begin{array}{l}\text { Quartile } 4(\mathrm{n}=141) \\
>3.4 \mathrm{~L}\end{array}$ & $P$ value \\
\hline Number of deaths & $1(0.8 \%)$ & $1(0.6 \%)$ & $2(1.8 \%)$ & $5(3.6 \%)$ & 0.203 \\
\hline Cause of death & & & & & 0.413 \\
\hline Postoperative complication & $0(0.0 \%)$ & $0(0.0 \%)$ & $0(0.0 \%)$ & $0(0.0 \%)$ & \\
\hline Cancer related & $0(0.0 \%)$ & $1(100.0 \%)$ & $1(50.0 \%)$ & $3(60.0 \%)$ & \\
\hline Non-cancer related & $0(0.0 \%)$ & $0(0.0 \%)$ & $0(0.0 \%)$ & $0(0.0 \%)$ & \\
\hline Uncertain & $1(100.0 \%)$ & $0(0.0 \%)$ & $1(50.0 \%)$ & $2(40.0 \%)$ & \\
\hline
\end{tabular}

Values are presented as $\mathrm{n}(\%)$. FVC, forced vital capacity.

Table S30 Mortality following video-assisted thoracic surgery lobectomy for lung cancer based on FEV1

\begin{tabular}{|c|c|c|c|c|c|}
\hline \multirow[t]{2}{*}{ Variables } & \multicolumn{5}{|c|}{ FEV1 } \\
\hline & $\begin{array}{c}\text { Quartile } 1(\mathrm{n}=122), \\
\leq 1.8 \mathrm{~L}\end{array}$ & $\begin{array}{c}\text { Quartile } 2(\mathrm{n}=167) \\
>1.8-2.3 \mathrm{~L}\end{array}$ & $\begin{array}{c}\text { Quartile } 3(n=127) \\
>2.3-2.7 \mathrm{~L}\end{array}$ & $\begin{array}{c}\text { Quartile } 4(\mathrm{n}=132) \text {, } \\
>2.7 \mathrm{~L}\end{array}$ & $P$ value \\
\hline Number of deaths & $1(0.8 \%)$ & $1(0.6 \%)$ & $1(0.8 \%)$ & $6(4.6 \%)$ & 0.057 \\
\hline Cause of death & & & & & 0.193 \\
\hline Postoperative complication & $0(0.0 \%)$ & $0(0.0 \%)$ & $0(0.0 \%)$ & $0(0.0 \%)$ & \\
\hline Cancer related & $1(100.0 \%)$ & $0(0.0 \%)$ & $0(0.0 \%)$ & $4(66.7 \%)$ & \\
\hline Non-cancer related & $0(0.0 \%)$ & $0(0.0 \%)$ & $0(0.0 \%)$ & $0(0.0 \%)$ & \\
\hline Uncertain & $0(0.0 \%)$ & $1(100.0 \%)$ & $1(100.0 \%)$ & $2(33.3 \%)$ & \\
\hline
\end{tabular}

Values are presented as $\mathrm{n}(\%)$. FEV1, forced expiratory volume in $1 \mathrm{~s}$. 
Table S31 Multivariate Cox regression results for overall survival based on PEF

\begin{tabular}{|c|c|c|c|}
\hline Variables & HR & $95 \% \mathrm{Cl}$ & $P$ value \\
\hline ASA score & & & 0.999 \\
\hline 1 & 1.000 & & \\
\hline ॥ & 0.000 & 0.000 & 0.960 \\
\hline III & 0.000 & 0.000 & 0.976 \\
\hline \multicolumn{4}{|l|}{ Age } \\
\hline$<60$ & 1.000 & & \\
\hline $60-$ & 0.720 & $0.159-3.262$ & 0.670 \\
\hline \multicolumn{4}{|l|}{ Gender } \\
\hline Male & 1.000 & & \\
\hline Female & 0.857 & $0.072-10.229$ & 0.903 \\
\hline \multicolumn{4}{|l|}{ BMI } \\
\hline$<24.0 \mathrm{~kg} / \mathrm{m}^{2}$ & 1.000 & & \\
\hline$\geq 24.0 \mathrm{~kg} / \mathrm{m}^{2}$ & 0.972 & $0.179-5.277$ & 0.974 \\
\hline \multicolumn{4}{|l|}{ Smoking } \\
\hline No & 1.000 & & \\
\hline Yes & 5.820 & $0.684-49.509$ & 0.107 \\
\hline \multicolumn{4}{|l|}{ Diabetes mellitus } \\
\hline No & 1.000 & & \\
\hline Yes & 0.000 & 0.000 & 0.955 \\
\hline \multicolumn{4}{|l|}{ Coronary heart disease } \\
\hline No & 1.000 & & \\
\hline Yes & 0.000 & 0.000 & 0.995 \\
\hline \multicolumn{4}{|l|}{ Intraoperative bleeding } \\
\hline$<50 \mathrm{~mL}$ & 1.000 & & \\
\hline $50 \mathrm{~mL}-$ & 8.747 & $0.852-89.843$ & 0.068 \\
\hline \multicolumn{4}{|l|}{ Length of operation } \\
\hline$<2 \mathrm{~h}$ & 1.000 & & \\
\hline $2 \mathrm{~h}-$ & 1.447 & $0.254-8.249$ & 0.677 \\
\hline NSCLC staging & & & 0.121 \\
\hline IA & 1.000 & & \\
\hline IB & 0.000 & 0.000 & 0.981 \\
\hline IIA & 0.000 & 0.000 & 0.980 \\
\hline IIB & 0.000 & 0.000 & 0.997 \\
\hline IIIA & 12.589 & $2.004-79.074$ & 0.007 \\
\hline Postoperative pathology & & & 0.999 \\
\hline Adenocarcinoma & 1.000 & & \\
\hline Squamous cell carcinoma & 0.000 & 0.000 & 0.961 \\
\hline Adenosquamous carcinoma & 0.000 & 0.000 & 0.990 \\
\hline PEF, L/s & & & 0.920 \\
\hline$\leq 3.3 \mathrm{~L} / \mathrm{s}$ & 0.000 & 0.000 & 0.926 \\
\hline$>3.3-4.4 \mathrm{~L} / \mathrm{s}$ & 0.451 & $0.037-5.503$ & 0.533 \\
\hline$>4.4-5.8 \mathrm{~L} / \mathrm{s}$ & 0.936 & $0.115-7.636$ & 0.951 \\
\hline$>5.8 \mathrm{~L} / \mathrm{s}$ & 1.000 & & \\
\hline
\end{tabular}

Results of binary logistics regression are presented as adjusted $\mathrm{HR}, 95 \% \mathrm{Cl}$ and $\mathrm{P}$ value. ASA, American Society of Anaesthesiologists; $\mathrm{BMI}$, body mass index; $\mathrm{Cl}$, confidence interval; HR, hazard ratio; NSCLC, non-small cell lung cancer; PEF, peak expiratory flow. 
Table S32 Multivariate Cox regression results for overall survival based on FVC

\begin{tabular}{|c|c|c|c|}
\hline Variables & $\mathrm{HR}$ & $95 \% \mathrm{Cl}$ & $P$ value \\
\hline ASA score & & & 0.999 \\
\hline I & 1.000 & & \\
\hline ॥ & 0.000 & 0.000 & 0.963 \\
\hline III & 0.000 & 0.000 & 0.975 \\
\hline \multicolumn{4}{|l|}{ Age } \\
\hline$<60$ & 1.000 & & \\
\hline $60-$ & 0.605 & $0.140-2.608$ & 0.500 \\
\hline \multicolumn{4}{|l|}{ Gender } \\
\hline Male & 1.000 & & \\
\hline Female & 0.481 & $0.032-7.282$ & 0.598 \\
\hline \multicolumn{4}{|l|}{ BMI } \\
\hline$<24.0 \mathrm{~kg} / \mathrm{m}^{2}$ & 1.000 & & \\
\hline$\geq 24.0 \mathrm{~kg} / \mathrm{m}^{2}$ & 0.611 & $0.118-3.155$ & 0.556 \\
\hline \multicolumn{4}{|l|}{ Smoking } \\
\hline No & 1.000 & & \\
\hline Yes & 4.829 & $0.625-37.325$ & 0.131 \\
\hline \multicolumn{4}{|l|}{ Diabetes mellitus } \\
\hline No & 1.000 & & \\
\hline Yes & 0.000 & 0.000 & 0.959 \\
\hline \multicolumn{4}{|l|}{ Coronary heart disease } \\
\hline No & 1.000 & & \\
\hline Yes & 0.000 & 0.000 & 0.997 \\
\hline \multicolumn{4}{|l|}{ Intraoperative bleeding } \\
\hline$<50 \mathrm{~mL}$ & 1.000 & & \\
\hline $50 \mathrm{~mL}-$ & 11.595 & $0.986-136.357$ & 0.051 \\
\hline \multicolumn{4}{|l|}{ Length of operation } \\
\hline$<2 \mathrm{~h}$ & 1.000 & & \\
\hline $2 \mathrm{~h}-$ & 1.598 & $0.284-9.000$ & 0.595 \\
\hline NSCLC staging & & & 0.072 \\
\hline IA & 1.000 & & \\
\hline IB & 0.000 & 0.000 & 0.975 \\
\hline IIA & 0.000 & 0.000 & 0.974 \\
\hline IIB & 0.000 & 0.000 & 0.997 \\
\hline IIIA & 22.032 & $2.784-174.356$ & 0.003 \\
\hline Postoperative pathology & & & 0.998 \\
\hline Adenocarcinoma & 1.000 & & \\
\hline Squamous cell carcinoma & 0.000 & 0.000 & 0.957 \\
\hline Adenosquamous carcinoma & 0.000 & 0.000 & 0.988 \\
\hline FVC, L & & & 0.825 \\
\hline$\leq 2.3 \mathrm{~L}$ & 0.257 & $0.014-4.837$ & 0.364 \\
\hline$>2.3-2.9 \mathrm{~L}$ & 0.481 & $0.023-9.868$ & 0.635 \\
\hline$>2.9-3.4 \mathrm{~L}$ & 0.339 & $0.014-7.990$ & 0.502 \\
\hline$>3.4 \mathrm{~L}$ & 1.000 & & \\
\hline
\end{tabular}

Results of binary logistics regression are presented as adjusted $\mathrm{HR}, 95 \% \mathrm{Cl}$ and $\mathrm{P}$ value. ASA, American Society of Anaesthesiologists; $\mathrm{BMI}$, body mass index; $\mathrm{Cl}$, confidence interval; FVC, forced vital capacity; HR, hazard ratio; NSCLC, non-small cell lung cancer. 
Table S33 Multivariate Cox regression results for overall survival based on FEV1

\begin{tabular}{|c|c|c|c|}
\hline Variables & $\mathrm{HR}$ & $95 \% \mathrm{Cl}$ & $P$ value \\
\hline ASA score & & & 0.999 \\
\hline I & 1.000 & & \\
\hline ॥ & 0.000 & 0.000 & 0.968 \\
\hline III & 0.000 & 0.000 & 0.977 \\
\hline \multicolumn{4}{|l|}{ Age } \\
\hline$<60$ & 1.000 & & \\
\hline $60-$ & 0.835 & $0.193-3.604$ & 0.809 \\
\hline \multicolumn{4}{|l|}{ Gender } \\
\hline Male & 1.000 & & \\
\hline Female & 1.092 & $0.064-18.546$ & 0.952 \\
\hline \multicolumn{4}{|l|}{ BMI } \\
\hline$<24.0 \mathrm{~kg} / \mathrm{m}^{2}$ & 1.000 & & \\
\hline$\geq 24.0 \mathrm{~kg} / \mathrm{m}^{2}$ & 0.703 & $0.129-3.837$ & 0.684 \\
\hline \multicolumn{4}{|l|}{ Smoking } \\
\hline No & 1.000 & & \\
\hline Yes & 4.917 & $0.640-37.806$ & 0.126 \\
\hline \multicolumn{4}{|l|}{ Diabetes mellitus } \\
\hline No & 1.000 & & \\
\hline Yes & 0.000 & 0.000 & 0.964 \\
\hline \multicolumn{4}{|l|}{ Coronary heart disease } \\
\hline No & 1.000 & & \\
\hline Yes & 0.000 & 0.000 & 0.996 \\
\hline \multicolumn{4}{|l|}{ Intraoperative bleeding } \\
\hline$<50 \mathrm{~mL}$ & 1.000 & & \\
\hline $50 \mathrm{~mL}-$ & 9.980 & $0.936-106.453$ & 0.057 \\
\hline \multicolumn{4}{|l|}{ Length of operation } \\
\hline$<2 \mathrm{~h}$ & 1.000 & & \\
\hline $2 \mathrm{~h}-$ & 1.745 & $0.307-9.922$ & 0.530 \\
\hline NSCLC staging & & & 0.170 \\
\hline IA & 1.000 & & \\
\hline IB & 0.000 & 0.000 & 0.973 \\
\hline IIA & 0.000 & 0.000 & 0.977 \\
\hline IIB & 0.000 & 0.000 & 0.997 \\
\hline IIIA & 11.694 & $1.743-78.475$ & 0.011 \\
\hline Postoperative pathology & & & 0.999 \\
\hline Adenocarcinoma & 1.000 & & \\
\hline Squamous cell carcinoma & 0.000 & 0.000 & 0.961 \\
\hline Adenosquamous carcinoma & 0.000 & 0.000 & 0.989 \\
\hline FEV1, L & & & 0.611 \\
\hline$\leq 1.8 \mathrm{~L}$ & 0.327 & $0.018-5.818$ & 0.446 \\
\hline$>1.8-2.3 \mathrm{~L}$ & 0.374 & $0.015-9.076$ & 0.546 \\
\hline$>2.3-2.7 \mathrm{~L}$ & 1.283 & $0.060-27.572$ & 0.873 \\
\hline$>2.7 \mathrm{~L}$ & 1.000 & & \\
\hline
\end{tabular}

Results of binary logistics regression are presented as adjusted $\mathrm{HR}, 95 \% \mathrm{Cl}$ and $\mathrm{P}$ value. ASA, American Society of Anaesthesiologists $\mathrm{BMI}$, body mass index; $\mathrm{Cl}$, confidence interval; FEV1, forced expiratory volume in $1 \mathrm{~s}$; HR, hazard ratio; NSCLC, non-small cell lung cancer. 